\title{
2-Regularized Nyquist Criterion in Linear Continuous-Time Periodic Systems and Its Implementation
}

$\operatorname{AUTHOR}(S)$ :

Zhou, Jun; Hagiwara, Tomomichi

\section{CITATION:}

Zhou, Jun ...[et al]. 2-Regularized Nyquist Criterion in Linear Continuous-Time Periodic Systems and Its Implementation. SIAM Journal on Control and Optimization 2005, 44(2): 618-645

ISSUE DATE:

2005-09-12

URL:

http://hdl.handle.net/2433/194119

RIGHT:

(C) 2005 Society for Industrial and Applied Mathematics 


\title{
2-REGULARIZED NYQUIST CRITERION IN LINEAR CONTINUOUS-TIME PERIODIC SYSTEMS AND ITS IMPLEMENTATION*
}

\author{
JUN ZHOU $^{\dagger}$ AND TOMOMICHI HAGIWARA ${ }^{\dagger}$
}

\begin{abstract}
First, by using the 2-regularized determinant technique for Hilbert-Schmidt operators, the computation formula, infinite-product convergence, and analyticity of the 2-regularized determinant of the modified harmonic state operator in finite-dimensional linear continuous-time periodic (FDLCP) systems are derived in this paper. Second, based on these results, a 2-regularized Nyquist criterion is established for asymptotic stability analysis of a class of FDLCP systems for the first time. Third, a numeric implementation algorithm for the 2-regularized Nyquist criterion is also proposed via the staircase truncation on the harmonic transfer operator of the FDLCP system concerned. Finally, to illustrate the results of this paper, asymptotic stability of the lossy Mathieu differential equation is investigated.
\end{abstract}

Key words. continuous-time periodic system, Nyquist criterion, determinant of HilbertSchmidt operators, implementation, convergence

AMS subject classifications. 43A32, 65N12, 65F40, 93D20

DOI. $10.1137 / \mathrm{S} 0363012902416900$

1. Introduction. Stability analysis is a difficult topic in finite-dimensional linear continuous-time periodic (FDLCP) systems [3], [8], [14], [19], [27], which are encountered in many engineering applications. For instance, the flapping dynamics of helicopter rotors [7], [26] and rolling motion of ships in waves [1] can be related to FDLCP models. Other examples include robot arms moving along periodic trajectories and electromechanical oscillation in AC generators [22]. Different types of (closed-loop) stability of FDLCP systems are discussed via various methods in the literature. Absolute stability of FDLCP systems with nonlinearities satisfying integral quadratic constraints is dealt with in [16] and [28] by the cutting plane algorithm and the Hamiltonian approach, respectively, while input/output stability and Youla-style parameterization of stabilizing controllers are discussed in [6] via the graph representation theory. As for asymptotic stability analysis of FDLCP systems, the Floquet theorem [18] completes the task by testing the eigenvalues of its monodromy matrix that is hard to find. Asymptotic stability has also been examined by a Lyapunov method [4] and the harmonic analysis [31]. Perturbation methods to study stability in FDLCP systems can be found in [20]. Nyquist-type stability criteria have also been considered in the FDLCP cases; for example, two generalized Nyquist criteria are suggested in [15] and [26]. The former is an integral-operator-based Nyquist criterion, while the latter is given in terms of the Hill-determinant of the infinite-dimensional harmonic transfer operator of an FDLCP system. However, due to the infinite-dimensionality and various convergence issues in the Hill-determinant, the validity of the latter generalized Nyquist criterion [26] remains as an open problem in general situations. Similar comments also apply to the former criterion. As for integral-operator modeling of periodic systems, we refer the readers to [2] for a general idea.

\footnotetext{
* Received by the editors November 1, 2002; accepted for publication (in revised form) March 10, 2005; published electronically September 12, 2005.

http://www.siam.org/journals/sicon/44-2/41690.html

${ }^{\dagger}$ Department of Electrical Engineering, Kyoto University, Kyotodaigaku-Katsura, Nishikyo-ku, Kyoto 615-8510, Japan (zhouj@kuee.kyoto-u.ac.jp, hagiwara@kuee.kyoto-u.ac.jp).
} 
A crucial observation for establishing Nyquist-type stability criteria in more general FDLCP systems is that the harmonic transfer operators of FDLCP systems are Hilbert-Schmidt operators under mild assumptions. Hence the validity of the 2regularized determinant on the harmonic transfer operators [5] can be justified. It should be pointed out that the harmonic transfer operators do not belong to the trace class [9] in general so that the standard operator determinant cannot be validated. Thus, developing a Nyquist-type criterion based on the 2-regularized determinant technique provides us with a natural and much stronger tool in stability analysis in the FDLCP field. In connection with the usual determinant defined on trace class operators, it is worth mentioning that in sampled-data systems, which are periodic (but not included in FDLCP systems) if the signal behavior both at the sampling instants and intersamples is considered, a Nyquist criterion regarding internal stability has been recovered with the transfer operator defined via lifting technique [13] under the assumption that the transfer operator is a trace class operator.

In this paper, using the 2-regularized determinant on Hilbert-Schmidt operators, we first derive some interesting analytic properties of the 2-regularized determinant of what we call the modified harmonic state operator of an FDLCP system. These results have not been explicitly discussed in the literature to the authors' best knowledge and constitute a significant contribution to this study. Second, based on these properties, a 2-regularized Nyquist criterion is established for asymptotic stability of a class of FDLCP systems. This Nyquist criterion is necessary and sufficient, and makes it possible for us to investigate the closed-loop asymptotic stability via the open-loop FDLCP system and the 2-regularized determinant of the corresponding harmonic return difference operator, similar to what we do in the LTI (linear timeinvariant) continuous-time case. In spite of the success that the criterion applies to a big class of practical FDLCP systems, however, it brings another problem. Namely, it is nontrivial to implement the criterion numerically because of the 2-regularized determinant on the infinite-dimensional harmonic return difference operator. To resolve the problem, the staircase truncation [30] is applied to the harmonic transfer operator. It is shown that under mild assumptions the truncation convergence can be ensured, and the 2-regularized Nyquist criterion can be implemented via only finitedimensional computations to any degree of accuracy, and the truncation size can be estimated readily through simple computations.

The following is the outline of this paper. Section 2 gives preliminaries to FDLCP systems, their harmonic state operators and transfer operators, the Toeplitz transformation of periodic functions, and operator determinants. Properties about the 2regularized determinants of the modified harmonic transfer operators are also derived. In section 3, the 2-regularized Nyquist criterion is established, while its implementation is considered via truncation in section 4 . The lossy Mathieu equation is studied to illustrate the results in section 5. Proofs of lemmas, if any, are given in appendices.

In this paper, $\|\cdot\|$ denotes the Euclidean norm of a vector and the norm of a matrix induced by this norm. $l_{2}$ is the set of all infinite-dimensional vectors $\underline{x}$ such that $\|\underline{x}\|_{l_{2}}^{2}:=\sum_{-\infty}^{+\infty}\left\|[\underline{x}]_{m}\right\|^{2}<\infty$, where $[\underline{x}]_{m}$ is the $m$ th (vector) entry of $\underline{x} .\|\cdot\|_{l_{2} / l_{2}}$ is the $l_{2}$-induced norm. $L_{2}[0, h]$ is the linear space of all vector measurable functions $x$ defined on $[0, h]$ such that $\|x(\cdot)\|_{L_{2}[0, h]}:=\left[\int_{0}^{h}\|x(t)\|^{2} d t\right]^{1 / 2}<\infty . F(\cdot) \in L_{2}[0, h]$ means that $F$ is an $h$-periodic matrix function, each element of which belongs to $L_{2}[0, h]$ when its domain is restricted to $[0, h]$. This expression is also used for other function sets defined over $[0, h]$. $\mathbf{C}$ is the field of all complex numbers, and $\mathbf{Z}$ is the ring of all integers. 
2. Preliminaries to FDLCP systems and operator determinants. In this section we first review facts about FDLCP systems [29], and then the 2-regularized determinant [5] for Hilbert-Schmidt operators. In particular, we derive properties about the harmonic state operator of FDLCP systems in the 2-regularized determinant sense.

Consider the FDLCP system given by

$$
G:\left\{\begin{array}{l}
\dot{x}=A(t) x+B(t) u \\
y=C(t) x
\end{array}\right.
$$

where $A(t), B(t)$, and $C(t)$ are $h$-periodically time-varying matrices. The transition matrix of (2.1) with the initial time $t_{0}$ is denoted by $\Phi\left(t, t_{0}\right)$. By the Floquet theorem [14], [18], if $A(t) \in L_{2}[0, h]$, then $\Phi\left(t, t_{0}\right)$ is continuous with respect to $t$ and has a Floquet factorization $\Phi\left(t, t_{0}\right)=P\left(t, t_{0}\right) e^{Q\left(t-t_{0}\right)}$, where $P\left(t, t_{0}\right)$ is absolutely continuous in $t$, nonsingular and $h$-periodic in $t$ and $t_{0}$, and $Q$ is a constant matrix. Moreover, the system is asymptotically stable if and only if the eigenvalues of $Q$ lie in the open left-half plane. Without loss of generality, we assume $t_{0}=0$.

Now we review the Toeplitz transformation of periodic functions. Expand $X(t) \in$ $L_{2}[0, h]$ to its Fourier series $\sum_{m=-\infty}^{+\infty} X_{m} e^{j m \omega_{h} t}$ with $\omega_{h}:=2 \pi / h$. The Toeplitz transformation on $X(t)$, denoted by $\mathcal{T}\{X(t)\}$, maps $X(t)$ onto a doubly infinitedimensional block Toeplitz operator [26] (or block Laurent operator [10, p. 564]) of the form

$$
\mathcal{T}\{X(t)\}:=\left[\begin{array}{ccccc}
\ddots & \vdots & \vdots & \vdots & \cdot \\
\cdots & X_{0} & X_{-1} & X_{-2} & \cdots \\
\cdots & X_{1} & X_{0} & X_{-1} & \cdots \\
\cdots & X_{2} & X_{1} & X_{0} & \cdots \\
\cdot & \vdots & \vdots & \vdots & \ddots
\end{array}\right]=: \underline{X} .
$$

We further define $\underline{A}:=\mathcal{T}\{A(t)\}, \underline{B}:=\mathcal{T}\{B(t)\}, \underline{C}:=\mathcal{T}\{C(t)\}, \underline{P}:=\mathcal{T}\{P(t, 0)\}$, $\underline{\hat{B}}:=\mathcal{T}\left\{P^{-1}(t, 0) B(t)\right\}, \underline{\hat{C}}:=\mathcal{T}\{C(t) P(t, 0)\}, \underline{Q}:=\operatorname{diag}[\ldots, Q, Q, Q, \ldots]$, and

$$
\underline{E}(s):=\operatorname{diag}\left[\ldots, \varphi_{-2}(s) I, \varphi_{-1}(s) I, \varphi_{0}(s) I, \varphi_{1}(s) I, \varphi_{2}(s) I, \ldots\right],
$$

where $\varphi_{m}(s):=s+j m \omega_{h}, m \in \mathbf{Z}, s \in \mathbf{C}$. It follows that $\underline{E}(s)=\underline{E}(j 0)+s \underline{I}$, where $\underline{I}:=\mathcal{T}\{I\}$.

We need the following function sets to validate the Fourier analysis and Toeplitz transformation operations involved (see [29] and [32] for details) and simplify our statements.

$$
\begin{aligned}
& L_{\mathrm{PCD}}[0, h]:=\left\{f(t): \begin{array}{l}
f(t) \text { is piecewise continuous and } \\
\text { differentiable at a.e. } t \in[0, h]
\end{array}\right\}, \\
& L_{\mathrm{PCC}}[0, h]:=\left\{f(t): \begin{array}{l}
f(t) \text { is piecewise continuous and its Fourier series } \\
\text { expansion is convergent to } f\left(t_{0}\right) \text { for a.e. } t_{0} \in[0, h]
\end{array}\right\}, \\
& L_{\mathrm{CAC}}[0, h]:=\left\{f(t): \begin{array}{l}
f(t) \text { is continuous and the Fourier series } \\
\text { expansion of } f(t) \text { is absolutely convergent }
\end{array}\right\} \subset L_{\mathrm{PCC}}[0, h] .
\end{aligned}
$$

Here PCD stands for piecewise continuous and differentiable, and PCC is short for piecewise continuous and convergent, while CAC is the abbreviation for continuous and absolutely convergent. 
Now we state the similarity transformation formulas and eigenvalues of FDLCP systems in terms of the Toeplitz transformation of the system matrices and the Floquet factorization of the transition matrix [29], [31]. To facilitate the statements, let $l_{E}:=$ $\left\{\underline{x} \in l_{2}: \underline{E}(j 0) \underline{x} \in l_{2}\right\}$. Then, $l_{E}$ is a proper subset of $l_{2}$ and dense in $l_{2}$ [29].

LEMMA 2.1. In the FDLCP system (2.1), assume that $A(t) \in L_{\mathrm{PCD}}[0, h]$ and $B(t), C(t) \in L_{\mathrm{PCC}}[0, h]$. Then, $l_{E}$ is $\underline{P}$ - and $\underline{P}^{-1}$-invariant. Also, the unbounded operators $\underline{P}(\underline{E}(j 0)-Q) \underline{P}^{-1}$ and $\underline{E}(j 0)-\underline{A}$ are densely defined on $l_{2}$ (or equivalently, well defined on the subset $l_{E} \subset l_{2}$ ) and coincide with each other:

$$
\underline{P}(\underline{E}(j 0)-\underline{Q}) \underline{P}^{-1}=\underline{E}(j 0)-\underline{A} .
$$

Moreover, it holds on the whole Hilbert space $l_{2}$ that $\underline{\hat{B}}=\underline{P}^{-1} \underline{B}$ and $\underline{\hat{C}}=\underline{C} \underline{P}$.

Furthermore, system (2.1) is asymptotically stable if and only if the set $\Lambda$ of all eigenvalues of $Q-\underline{E}(j 0)$, i.e., $\Lambda=\left\{\lambda(Q)+j m \omega_{h}: m \in \mathbf{Z}\right\}$, lies in the open left-half plane. It is also true that $\Lambda=\Lambda_{A}$ where $\Lambda_{A}$ is the set of all eigenvalues of $\underline{A}-\underline{E}(j 0)$. In the above, $\lambda(\cdot)$ denotes the set of all eigenvalues of the matrix $(\cdot)$.

Now we introduce the harmonic transfer operator [26] of system (2.1) given by

$$
\underline{G}(s):=\underline{C}(\underline{E}(s)-\underline{A})^{-1} \underline{B}
$$

in which $\underline{A}-\underline{E}(s)$ is called the harmonic state operator of system (2.1). In view of (2.4) in Lemma 2.1, $Q-\underline{E}(s)$ is called the Floquet state operator of (2.1) to distinguish it from $\underline{A}-\underline{E}(s)$. When $\underline{E}^{-1}(s)$ exists, $\underline{I}-\underline{E}^{-1}(s) \underline{A}$ (respectively, $\underline{I}-\underline{E}^{-1}(s) Q$ ) will be called the modified harmonic state operator (respectively, the modified Floquet state operator) of system (2.1).

Now we consider a domain $\Omega \subset \mathbf{C}$ and assume that $s \in \Omega$. Let us further give assumptions $\mathrm{A} 1$ and $\mathrm{A} 2$ about $\Omega$ to facilitate our statements.

A1 The domain $\Omega$ is closed and has a simple closed boundary, denoted by $\partial \Omega$, and thus is a simply connected domain on $\mathbf{C}$. Also, it holds that

$$
|\operatorname{Im}(s)|<K_{\Omega}:=\omega_{h} \quad(\forall s \in \Omega) .
$$

Furthermore, $\underline{E}(s)-Q$ is an invertible mapping from $l_{E}$ to $l_{2}$ for each $s \in \partial \Omega$. A2 On the domain $\Omega, \underline{E}(s)$ is an invertible mapping from $l_{E}$ to $l_{2}$.

Note that the last assumption of A1 is satisfied if and only if $\partial \Omega$ contains no points in $\Lambda$, while A2 is satisfied if and only if $\Omega$ does not contain any points in $\Gamma:=\left\{j m \omega_{h}\right.$ : $m \in \mathbf{Z}\}$. Hence, relation (2.4) tells us that

$$
\underline{P}(\underline{E}(s)-\underline{Q})^{-1} \underline{P}^{-1}=(\underline{E}(s)-\underline{A})^{-1}
$$

for all $s \in \Omega \backslash \Lambda$. That is, $\underline{E}(s)-\underline{A}$ is an invertible mapping from $l_{E}$ to $l_{2}$ for each $s \in \Omega \backslash \Lambda$. (2.7) says that the harmonic transfer operator $\underline{G}(s)$ is well defined on $l_{2}$ for all $s \in \Omega \backslash \Lambda$. Lemma 2.2 gives basic facts about $\underline{G}(s)$ that play a key role in developing the Nyquist criterion.

LEMMA 2.2. In the FDLCP system (2.1), let $A(t) \in L_{\mathrm{PCD}}[0, h]$ and $B(t), C(t) \in$ $L_{\mathrm{PCC}}[0, h]$. Assume that the domain $\Omega$ satisfies A1. Then for each $s \in \Omega \backslash \Lambda,(\underline{E}(s)-$ $\underline{Q})^{-1} \in \mathcal{C}_{2}\left(l_{2}\right)$, and thus $\underline{G}(s) \in \mathcal{C}_{2}\left(l_{2}\right)$. Furthermore, $\|\underline{G}(s)\|_{2}$ has a uniform upper bound over $s \in \partial \Omega$.

Now we introduce the 2-regularized determinant of Hilbert-Schmidt operators and derive some properties about the 2-regularized determinant of the modified harmonic transfer operators. 
Let $\lambda_{i}(A)$ denote the $i$ th eigenvalue of a linear compact operator $A: l_{2} \rightarrow l_{2}$, and $s_{i}(A):=\left(\lambda_{i}\left(A^{*} A\right)\right)^{1 / 2}$ be its $i$ th singular value. For $p=1$ and 2 , the set of all compact operators $A: l_{2} \rightarrow l_{2}$ satisfying $\|A\|_{p}:=\left(\sum_{i} s_{i}(A)^{p}\right)^{1 / p}<\infty$ is denoted by $\mathcal{C}_{1}\left(l_{2}\right)$ and $\mathcal{C}_{2}\left(l_{2}\right)$, respectively. In particular, the operators in $\mathcal{C}_{1}\left(l_{2}\right)$ are called trace class operators while those in $\mathcal{C}_{2}\left(l_{2}\right)$ are called Hilbert-Schmidt operators [5]. Clearly, $\mathcal{C}_{1}\left(l_{2}\right) \subset \mathcal{C}_{2}\left(l_{2}\right)$. For $A \in \mathcal{C}_{1}\left(l_{2}\right)$, the operator trace and determinant below are well defined in the sense that the infinite series and product converge

$$
\operatorname{tr}(A):=\sum \lambda_{i}(A), \quad \operatorname{det}(I+A):=\prod\left(1+\lambda_{i}(A)\right) .
$$

Note that for $A \in \mathcal{C}_{2}\left(l_{2}\right), R_{2}(A):=(I+A) \exp \{-A\}-I \in \mathcal{C}_{1}\left(l_{2}\right)$. Thus, it is justified to define the determinant of $I+R_{2}(A)$ in the sense of $(2.8)$, denoted by $\operatorname{det}_{2}(I+A):=\operatorname{det}\left(I+R_{2}(A)\right)$, which is called the 2-regularized determinant of $I+A$. For our aim, assume that $B \in \mathcal{C}_{2}\left(l_{2}\right)$. Then

$$
\begin{gathered}
\operatorname{det}_{2}(I+A)=\prod\left[\left(1+\lambda_{i}(A)\right) \exp \left(-\lambda_{i}(A)\right)\right], \\
\operatorname{det}_{2}(I+A) \operatorname{det}_{2}(I+B)=\operatorname{det}_{2}[(I+A)(I+B)] \exp \{\operatorname{tr}(A B)\} .
\end{gathered}
$$

By Proposition 1.3 of [9, p. 98], if $A \in \mathcal{C}_{2}\left(l_{2}\right)$ and $B$ and $C$ are bounded linear operators on $l_{2}$, then $B A C$ belongs to $\mathcal{C}_{2}\left(l_{2}\right)$ and $\|B A C\|_{2} \leq\|B\|_{l_{2} / l_{2}}\|A\|_{2}\|C\|_{l_{2} / l_{2}}$. Moreover, Theorem 3.1 of $[9$, p. 43$]$ says that $A B$ and $B A$ have the same nonzero eigenvalues with multiplicity taken into account, i.e., $\operatorname{det}_{2}(I+A B)=\operatorname{det}_{2}(I+B A)$.

When establishing a Nyquist-type criterion for FDLCP systems, one might be tempted to talk about some sort of determinant about the harmonic state operator $\underline{A}-\underline{E}(s)$ or the Floquet state operator $\underline{Q}-\underline{E}(s)$ as in the LTI continuous-time case. However, such a determinant notion for these unbounded operators is not readily available in the literature. The following lemma will be a key to get around the difficulty, in which the 2-regularized determinant of these operators premultiplied by $-\underline{E}^{-1}(s)$ (i.e., the modified harmonic and Floquet state operators) are considered.

LEMMA 2.3. In the FDLCP system (2.1), let $A(t) \in L_{\mathrm{CAC}}[0, h] \cap L_{\mathrm{PCD}}[0, h]$. Assume that the domain $\Omega$ satisfies A1 and A2. Then for each $s \in \Omega, E^{-1}(s) \in$ $\mathcal{C}_{2}\left(l_{2}\right)$, and thus $\underline{E}^{-1}(s) \underline{A} \in \mathcal{C}_{2}\left(l_{2}\right), \underline{E}^{-1}(s) \underline{Q} \in \mathcal{C}_{2}\left(l_{2}\right)$. In particular, $\left\|\underline{E}^{-1}(s)\right\|_{2}$ has a uniform upper bound over $s \in \Omega$. Furthermore,

$$
\operatorname{det}_{2}\left[\underline{I}-\underline{E}^{-1}(s) \underline{A}\right]=g_{A}(s) \operatorname{det}_{2}\left[\underline{I}-\underline{E}^{-1}(s) \underline{Q}\right],
$$

where the function $g_{A}(s)$ does not vanish for each $s \in \Omega$ and is analytic over $\Omega$. Also, $\underline{I}-\underline{E}^{-1}(s) \underline{A}$ is invertible for each $s \in \Omega$, and the inverse of $\underline{I}-\underline{E}^{-1}(s) \underline{A}$ is bounded on $l_{2}$.

Lemma 2.4. Let $\lambda_{k}(Q)$ denote the $k$ th eigenvalue of the $n \times n$ matrix $Q$. If the domain $\Omega$ satisfies $\mathrm{A} 1$ and $\mathrm{A} 2$, then the function

$$
\begin{aligned}
f_{Q}(s) & :=\operatorname{det}_{2}\left[\underline{I}-\underline{E}^{-1}(s) \underline{Q}\right] \\
& =\prod_{k=1}^{n} \prod_{m=-\infty}^{\infty}\left(1-\frac{\lambda_{k}(Q)}{s+j m \omega_{h}}\right) \exp \left\{\frac{\lambda_{k}(Q)}{s+j m \omega_{h}}\right\}
\end{aligned}
$$

is analytic on $\Omega$, which has a zero at each point $\lambda_{k}(Q)-j m \omega_{h}, k=1,2, \ldots, n$, and $m \in \mathbf{Z}$ (i.e., counted up to multiplicity), and has no other zeros on the complex plane. 
Remark 1. Lemma 2.4 says that the set of all zeros of $f_{Q}(s)$ is equal to the set $\Lambda$ of all the eigenvalues of $Q-\underline{E}(j 0)$. This, together with Lemma 2.1, tells us that asymptotic stability of an FDLCP system can be reflected by the function $f_{Q}(s)$. This is the starting point of establishing a generalized Nyquist criterion via the 2regularized determinant approach. To relate $f_{Q}(s)$ to its closed-loop counterpart via the 2-regularized determinant of the harmonic return differential operator will be the task in the following section, by which a Nyquist criterion of FDLCP systems will be derived in a similar fashion to the corresponding result in the LTI continuous-time systems.

In the following, $\partial \Omega$ will be chosen to form a Nyquist contour $N_{r}$, which needs to directly pass through the origin or to include the origin in the interior of the region enclosed by $N_{r}$ (that is, to bypass the origin if there are any eigenvalues of $Q-\underline{E}(j 0)$ at the origin). However, such an $\Omega$ violates the assumption A2, since $0 \in \Gamma$. To surmount this problem, we introduce a shift factor $\rho>0$ to $s$ and replace assumption A2 by the following assumption.

A2 ${ }^{\prime}$ On the domain $\Omega, \underline{E}(s+\rho)(=\underline{E}(s)+\rho \underline{I})$ is an invertible mapping from $l_{E}$ to $l_{2}$.

As we shall see later, assumption $\mathrm{A} 2^{\prime}$ can in fact be essentially simplified to the condition $\rho>0$ in our context due to the specific choice of the domain $\Omega$ given later. The introduction of such $\rho>0$ is crucial only in the FDLCP setting exactly because $\underline{E}(s+\rho)$ is noninvertible at $s=0$ if $\rho=0$; this noninvertibility causes a problem when we try to deal with the 2-regularized determinant of $\underline{I}-\underline{E}^{-1}(s) \underline{A}$. It is easy to see that such a difficulty does not exist in the LTI continuous-time case since in that case $\operatorname{det}(s I-A)$ can be considered directly and $s$ need not be inverted (recall the paragraph just before Lemma 2.3).

Remark 2. Once we introduce $\rho>0$, we consider $\operatorname{det}_{2}\left[\underline{I}-\underline{E}^{-1}(s+\rho)(\underline{A}+\rho \underline{I})\right]$ and $\operatorname{det}_{2}\left[\underline{I}-\underline{E}^{-1}(s+\rho)(\underline{Q}+\rho \underline{I})\right]$ instead of similar relations in Lemmas 2.3 and 2.4 . Hence, what we indeed employ in the subsequent arguments is the accordingly modified versions of Lemmas 2.3 and 2.4. Note that A1 is not affected by this shift factor. Thus, Lemma 2.3 still holds even if A2 is replaced by A2', provided that $\underline{E}(s), g_{A}(s), \underline{A}$, and $Q$ are also replaced by $\underline{E}(s+\rho), g_{A+\rho I}(s+\rho), \underline{A}+\rho \underline{I}$, and $Q+\rho \underline{I}$, respectively. To facilitate the following descriptions, we will refer to this modified result as Lemma $2.3^{\prime}$. Similarly, Lemma 2.4 holds true when A2 is replaced by A2 ${ }^{\prime}$ if $\underline{E}(s), f_{Q}(s), \underline{Q}, \lambda_{k}(Q)$, and $j m \omega_{h}$ are replaced by $\underline{E}(s+\rho), f_{Q+\rho I}(s+\rho), Q+\rho \underline{I}, \lambda_{k}(Q+\rho I)$, and $\bar{\rho}+j m \omega_{h}$, respectively. This modified result will be referred to as Lemma $2.4^{\prime}$. Moreover, it is obvious that the set of zeros of $f_{Q+\rho I}(s+\rho)$ equals that of the set of zeros of $f_{Q}(s)$. Clearly, these modified results can be validated even if condition (2.6) is removed from A1, because of the periodicity of $\underline{E}(s)$.

It is worth noticing that the introduction of the shift factor $\rho>0$ does not cause any approximation effect at all on the stability analysis. This is because the invertibility of $\underline{I}-\underline{E}^{-1}(s+\rho)(\underline{A}+\rho \underline{I})$ is equivalent to that of $\underline{E}^{-1}(s+\rho)\left\{\underline{I}-\underline{E}^{-1}(s+\right.$ $\rho)(\underline{A}+\rho \underline{I})\}=\underline{E}(s)-\underline{A}$.

3. 2-Regularized Nyquist stability criterion. In this section, we develop a Nyquist criterion by the 2-regularized determinant for stability analysis of the closedloop FDLCP system when an output feedback is introduced. In system (2.1), let $A(t), B(t), C(t) \in L_{\mathrm{CAC}}[0, h] \cap L_{\mathrm{PCD}}[0, h]$, and an $h$-periodically time-varying output feedback $u=-K(t) y+v$ is introduced. This leads to the closed-loop FDLCP system 
described by

$$
G_{c}:\left\{\begin{array}{l}
\dot{x}=A_{c}(t) x+B(t) v, \\
y=C(t) x
\end{array}\right.
$$

Here $v$ is a new reference input and it is assumed that $K(t) \in L_{\mathrm{CAC}}[0, h] \cap L_{\mathrm{PCD}}[0, h]$. Clearly, $A_{c}(t):=A(t)-B(t) K(t) C(t) \in L_{\mathrm{CAC}}[0, h] \cap L_{\mathrm{PCD}}[0, h]$. These assumptions about $A(t), B(t), C(t)$, and $K(t)$ ensure that the Toeplitz transformation and Lemmas 2.1-2.4 (see also Remark 2) apply to both the open- and closed-loop FDLCP systems.

Now such a question emerges, In what way can one claim asymptotic stability of the closed-loop system $G_{c}$ by observing the open-loop harmonic transfer operator?

3.1. 2-Regularized determinant relation between open- and closed-loop modified harmonic state operators. As known in the LTI case, one must obtain the relationship between the open- and closed-loop pole polynomials before claiming the Nyquist criterion. The aim of this subsection is to get a similar relationship in the FDLCP case, where Lemma 2.4 and Remark 2 suggest that $f_{Q+\rho I}(s+\rho)=$ $\operatorname{det}_{2}\left[\underline{I}-\underline{E}^{-1}(s+\rho)(\underline{Q}+\rho \underline{I})\right]$, and the corresponding closed-loop counterpart plays the role of pole polynomials. To derive such a relationship, let us define $\underline{K}=\mathcal{T}\{K(t)\}$. Then it follows from Lemma 2.2 that for each $s \in \Omega \backslash \Lambda, \underline{K} \underline{G}(s)$ is well defined and belongs to $\mathcal{C}_{2}\left(l_{2}\right)$. Therefore, it makes sense to talk about the $\operatorname{det}_{2}$ of the return difference operator $\underline{I}+\underline{K} \underline{G}(s)$ for each $s \in \Omega \backslash \Lambda$. Noting that $\underline{E}(s+\rho)$ is invertible for all $s \in \Omega$ by $\mathrm{A}^{\prime}$, we compute $\operatorname{det}_{2}$ of $\underline{I}+\underline{K} \underline{G}(s)$ as

$$
\begin{aligned}
\operatorname{det}_{2}[\underline{I}+\underline{K} \underline{G}(s)] & =\operatorname{det}_{2}\left[\underline{I}+(\underline{E}(s)-\underline{A})^{-1} \underline{B} \underline{K} \underline{C}\right] \\
& =\operatorname{det}_{2}\left[\underline{I}+\left(\underline{I}-\underline{E}^{-1}(s+\rho)(\underline{A}+\rho \underline{I})\right)^{-1} \underline{E}^{-1}(s+\rho) \underline{B} \underline{K} \underline{C}\right] \\
& =\operatorname{det}_{2}\left[\left(\underline{I}-\underline{E}^{-1}(s+\rho)(\underline{A}+\rho \underline{I})\right)^{-1}\left(\underline{I}-\underline{E}^{-1}(s+\rho)\left(\underline{A}_{c}+\rho \underline{I}\right)\right)\right],
\end{aligned}
$$

where $\underline{A}_{c}:=\underline{A}-\underline{B} \underline{K} \underline{C}$. Note that $\underline{A}-\underline{B} \underline{K} \underline{C}=\mathcal{T}\left\{A_{c}(t)\right\}$ holds since $B(t), K(t)$, and $C(t)$ belong to $L_{\mathrm{CAC}}[0, h][17]$. In (3.2), we used the facts that $(\underline{E}(s)-\underline{A})^{-1} \in \mathcal{C}_{2}\left(l_{2}\right)$ for each $s \in \Omega \backslash \Lambda$ and that $\underline{K} \underline{C}$ is bounded on $l_{2}$, where the first fact can be shown by (2.7) and Lemma 2.2 .

Before we expand (3.2) via (2.10), we must show that the $\operatorname{det}_{2}$ 's of $\left(\underline{I}-\underline{E}^{-1}(s+\right.$ $\rho)(\underline{A}+\rho \underline{I}))^{-1}$ and $\underline{I}-\underline{E}^{-1}(s+\rho)\left(\underline{A}_{c}+\rho \underline{I}\right)$ are well defined for all $s \in \Omega \backslash \Lambda$. To this end, let us define the infinite-dimensional matrix $\underline{M}(s+\rho)$ such that for each $s \in \Omega \backslash \Lambda$, it holds that

$$
\left(\underline{I}-\underline{E}^{-1}(s+\rho)(\underline{A}+\rho \underline{I})\right)(\underline{I}+\underline{M}(s+\rho))=\underline{I} .
$$

It follows easily that $\underline{M}(s+\rho)$ is indeed given by

$$
\underline{M}(s+\rho)=\underline{E}^{-1}(s+\rho)(\underline{A}+\rho \underline{I})\left(\underline{I}-\underline{E}^{-1}(s+\rho)(\underline{A}+\rho \underline{I})\right)^{-1} .
$$

Then the fact that $\underline{E}^{-1}(s+\rho) \in \mathcal{C}_{2}\left(l_{2}\right)$ and that $(\underline{A}+\rho \underline{I})\left(\underline{I}-\underline{E}^{-1}(s+\rho)(\underline{A}+\rho \underline{I})\right)^{-1}$ is bounded on $l_{2}$ (by Lemma 2.3) implies that $\underline{M}(s+\rho) \in \mathcal{C}_{2}\left(l_{2}\right)$. Similarly, for each $s \in \Omega, \underline{E}^{-1}(s+\rho)\left(\underline{A}_{c}+\rho \underline{I}\right)$ and $\underline{E}^{-1}(s+\rho)(\underline{A}+\rho \underline{I})$ belong to $\mathcal{C}_{2}\left(l_{2}\right)$. Thus it makes sense to deal with the $\operatorname{det}_{2}$ 's of $\left(\underline{I}-\underline{E}^{-1}(s+\rho)(\underline{A}+\rho \underline{I})\right)^{-1}, \underline{I}-\underline{E}^{-1}(s+\rho)\left(\underline{A}_{c}+\rho \underline{I}\right)$, and $\underline{I}-\underline{E}^{-1}(s+\rho)(\underline{A}+\rho \underline{I})$ on $\Omega \backslash \Lambda$, separately.

Now taking the $\operatorname{det}_{2}$ on both sides of (3.3), we obtain

$$
\begin{aligned}
& \operatorname{det}_{2}\left[\underline{I}-\underline{E}^{-1}(s+\rho)(\underline{A}+\rho \underline{I})\right] \\
& =\frac{\exp \left\{-\operatorname{tr}\left(\underline{E}^{-1}(s+\rho)(\underline{A}+\rho \underline{I}) \underline{M}(s+\rho)\right)\right\}}{\operatorname{det}_{2}[\underline{I}+\underline{M}(s+\rho)]} .
\end{aligned}
$$


Then expanding the $\operatorname{det}_{2}$ of (3.2) via (2.10) and using (3.4), we have

$$
\begin{aligned}
\operatorname{det}_{2}[\underline{I}+\underline{K} \underline{G}(s)]= & \operatorname{det}_{2}\left[\left(\underline{I}-\underline{E}^{-1}(s+\rho)(\underline{A}+\rho \underline{I})\right)^{-1}\right] \\
& \times \operatorname{det}_{2}\left[\underline{I}-\underline{E}^{-1}(s+\rho)\left(\underline{A}_{c}+\rho \underline{I}\right)\right] \\
& \times \exp \left\{\operatorname{tr}\left(\underline{M}(s+\rho) \underline{E}^{-1}(s+\rho)\left(\underline{A}_{c}+\rho \underline{I}\right)\right)\right\} \\
= & \exp \{\Delta(s+\rho)\} \frac{\operatorname{det}_{2}\left[\underline{I}-\underline{E}^{-1}(s+\rho)\left(\underline{A}_{c}+\rho \underline{I}\right)\right]}{\operatorname{det}_{2}\left[\underline{I}-\underline{E}^{-1}(s+\rho)(\underline{A}+\rho \underline{I})\right]},
\end{aligned}
$$

where the scalar function $\Delta(s+\rho)$ is given by

$$
\begin{aligned}
\Delta(s+\rho): & :=\operatorname{tr}\left(\underline{M}(s+\rho) \underline{E}^{-1}(s+\rho)\left(\underline{A}_{c}+\rho \underline{I}\right)\right)-\operatorname{tr}\left(\underline{M}(s+\rho) \underline{E}^{-1}(s+\rho)(\underline{A}+\rho \underline{I})\right) \\
(3.6) & =-\operatorname{tr}\left(\underline{E}^{-1}(s+\rho)(\underline{A}+\rho \underline{I})(\underline{E}(s)-\underline{A})^{-1} \underline{B} \underline{K} \underline{C}\right) .
\end{aligned}
$$

3.2. Nyquist contour and Nyquist locus. Before leading the above arguments to a Nyquist stability criterion, we need to describe in what way an appropriate Nyquist contour, i.e., $\partial \Omega$, should be taken and how the corresponding Nyquist locus should be plotted in the 2-regularized determinant sense.

First let us see how an appropriate Nyquist contour should be taken.

To this purpose, we mention some facts about the eigenvalues of $Q-\underline{E}(j 0)$ (or equivalently the operator $\underline{A}-\underline{E}(j 0)$ ), which are given in Lemma 2.1. First, all the eigenvalues of $Q-\underline{E}(j 0)$ are located in a vertical strip region parallel to the imaginary axis; second, the eigenvalues distribution pattern in the horizontal strip

$$
\mathbf{C}_{F}:=\left\{s \in \mathbf{C}:-\omega_{h} / 2<\operatorname{Im}(s) \leq \omega_{h} / 2\right\},
$$

which is called the fundamental strip [26], unfolds itself vertically to both $-j \infty$ and $j \infty$ with the period $j \omega_{h}$. In other words, if we can understand the eigenvalue distribution pattern in $\mathbf{C}_{F}$, then the whole eigenvalue structure of $Q-\underline{E}(j 0)$ is clarified. Based on this, a possible Nyquist contour would be the boundary of the right-half fundamental strip of $\mathbf{C}_{F}$, i.e., $\left\{s: \operatorname{Re}(s) \geq 0, s \in \mathbf{C}_{F}\right\}$. However, since $\underline{G}(s)$ is not well defined for $s \in \Lambda$, the actual Nyquist contour should avoid going through these points in $\Lambda$. Hence the Nyquist contour $N_{r}$ shown in Figure 3.1 is taken. In Figure 3.1 the crosses ( $\times$ 's) denote possible eigenvalues of the open-loop operator $\underline{Q}-\underline{E}(j 0)$ on the boundary of the right-hand half of $\mathbf{C}_{F}$. It should be stressed that the Nyquist contour $N_{r}$ bypasses the eigenvalues of $Q-\underline{E}(j 0)$ on the imaginary axis with $-\omega_{h} / 2<\operatorname{Im}(\lambda)<\omega_{h} / 2$, if any, from the left-hand side while other eigenvalues on the boundary of the right-half fundamental strip, if any, from the upper-side, via a semicircle with the radius $r$ that is small enough. Thus, if $Q-\underline{E}(j 0)$ has eigenvalues on the imaginary axis such that $-\omega_{h} / 2<\operatorname{Im}(\lambda) \leq \omega_{h} / 2$, they are to be included in the interior of the region enclosed by $N_{r}$. Now let us assume that the right edge of $N_{r}$ is far enough from the imaginary axis so that there are no eigenvalues of $\underline{Q}-\underline{E}(j 0)$ on it, and finally, let us define the domain $\Omega$ as the union of the Nyquist contour $N_{r}$ and the interior of the region enclosed by $N_{r}$. Then, it is obvious that $\Omega$ satisfies A1 and A2' whenever $\rho>r \geq 0$ and $K_{\Omega}>\omega_{h} / 2+r$. Hence the arguments from (3.2) to (3.6) are validated for such a $\rho$.

Next let us see how the corresponding Nyquist locus can be plotted.

Now segment the Nyquist contour $N_{r}$ given in Figure 3.1 into four pieces $N_{a b}$, $N_{b c}, N_{c d}$, and $N_{d a}$ in the obvious fashion and note the following observations. First, since $\operatorname{det}_{2}[\underline{I}+\underline{K G}(s)]$ and $\Delta(s+\rho)$ are $j \omega_{h}$-periodic in the frequency domain, the plot of $\operatorname{det}_{2}[\underline{I}+\underline{K} \underline{G}(s)] \exp \{-\Delta(s+\rho)\}$ corresponding to $N_{a b}$ will form a closed curve. 


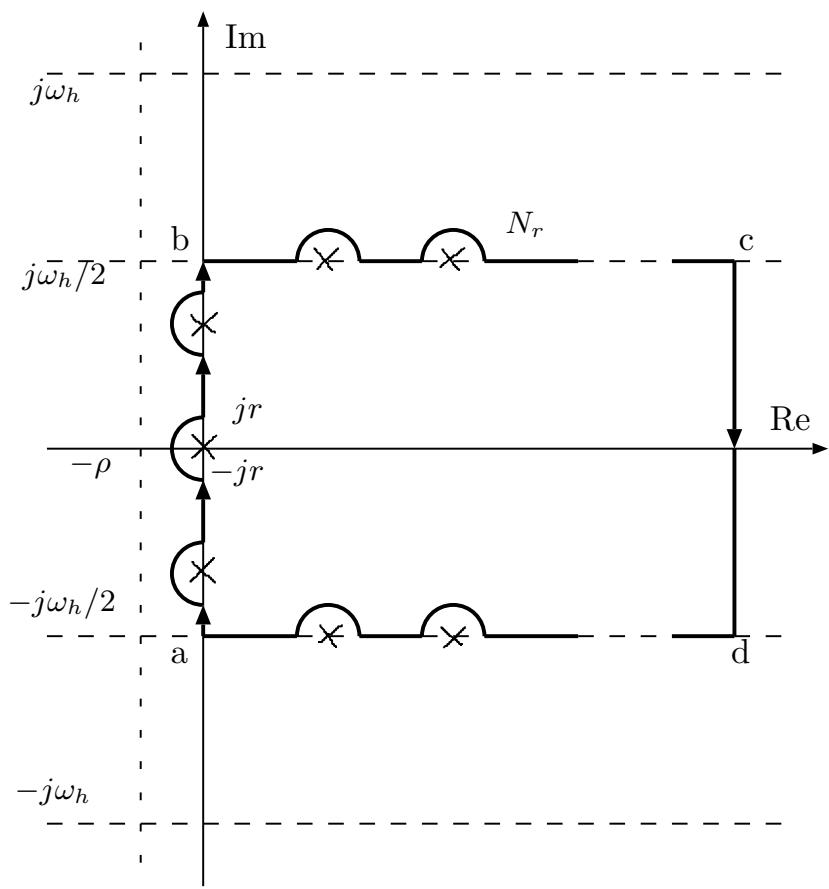

FIG. 3.1. Nyquist contour $N_{r}$.

Second, for each $s \in N_{b c}$, there is a corresponding complex number $\tilde{s}=s-j \omega_{h} \in$ $N_{d a}$ such that $\operatorname{det}_{2}[\underline{I}+\underline{K} \underline{G}(s)] \exp \{-\Delta(s+\rho)\}=\operatorname{det}_{2}[\underline{I}+\underline{K} \underline{G}(\tilde{s})] \exp \{-\Delta(\tilde{s}+$ $\rho)\}$ due to the same periodicity. The only difference between the plot of $\operatorname{det}_{2}[\underline{I}+$ $\underline{K} \underline{G}(s)] \exp \{-\Delta(s+\rho)\}$ corresponding to $N_{b c}$ and that corresponding to $N_{d a}$ is that these two plots are drawn in just opposite directions. To facilitate the discussions, we assume that $N_{b c}$ and $N_{d a}$ are taken in such a way that they bypass closed-loop eigenvalues, if any, in a similar fashion to what is done for open-loop eigenvalues. Clearly, this requirement on $N_{b c}$ and $N_{d a}$ can always be satisfied, in principle, and brings no essential difficulty to validate the second assertion we just claimed while keeping $\mathrm{A} 2^{\prime}$. Third, if $\operatorname{Re}(s)$ is made large enough for $s \in N_{c d}$ (i.e., the Nyquist contour $N_{r}$ is extended to the right far enough so that it encloses all the unstable closed-loop eigenvalues on the fundamental strip), $\operatorname{det}_{2}[\underline{I}+\underline{K} \underline{G}(s)] \exp \{-\Delta(s+\rho)\} \rightarrow 1$ for each $s \in N_{c d}$. These facts indicate that the plot segment of $\operatorname{det}_{2}[\underline{I}+\underline{K} \underline{G}(s)] \exp \{-\Delta(s+\rho)\}$ corresponding to $N_{b c}, N_{c d}$, and $N_{d a}$ neither goes through the origin nor contributes to encirclements around the origin. In other words, to investigate the encirclements of $\operatorname{det}_{2}[\underline{I}+\underline{K} \underline{G}(s)] \exp \{-\Delta(s+\rho)\}$ around the origin on $N_{r}$, it is enough to see the plot of $\operatorname{det}_{2}[\underline{I}+\underline{K} \underline{G}(s)] \exp \{-\Delta(s+\rho)\}$ corresponding to $N_{a b}$. In view of this, the plot $\operatorname{det}_{2}[\underline{I}+\underline{K} \underline{G}(s)] \exp \{-\Delta(s+\rho)\}: N_{a b} \rightarrow \mathbf{C}$ is called the Nyquist locus of (2.1) when $s \in N_{a b}$ moves in the clockwise direction with respect to $N_{r}$.

3.3. 2-Regularized Nyquist stability criterion. On the basis of arguments in the two preceding subsections, we are ready to show the 2-regularized Nyquist criterion, which is the main result of this paper.

TheOREM 3.1. Assume that $A(t), B(t), C(t)$ of the FDLCP system (2.1) and the feedback matrix $K(t)$ belong to $L_{\mathrm{CAC}}[0, h] \cap L_{\mathrm{PCD}}[0, h]$. Let $n_{\mathrm{us}}$ denote the number 
of the unstable eigenvalues of the open-loop Floquet state operator $\underline{Q}-\underline{E}(j 0)$ (or equivalently, the harmonic state operator $\underline{A}-\underline{E}(j 0))$ in the fundamental strip $\mathbf{C}_{F}$ defined in (3.7) counted according to their multiplicity. Take an arbitrary positive number $\rho$ and a sufficiently small number $r$ according to the open-loop eigenvalue condition of $Q-\underline{E}(j 0)$ on the imaginary axis such that $\rho>r \geq 0$, and then consider the harmonic transfer operator $G(s)$ and the scalar function $\Delta(s+\rho)$ defined in (3.6). Then, the closed-loop system $G_{c}$ is asymptotically stable if and only if the Nyquist locus, $\operatorname{det}_{2}[\underline{I}+\underline{K} \underline{G}(s)] \exp \{-\Delta(s+\rho)\}: N_{a b} \rightarrow \mathbf{C}$, vanishes nowhere on $N_{a b}$ and encircles the origin $n_{\mathrm{us}}$ times in the counterclockwise sense.

Proof. Under the given assumptions on the system matrices and the feedback gain matrix, Lemma 2.3' stated in Remark 2 ensures a modified version of (2.11), which reads

$$
\operatorname{det}_{2}\left[\underline{I}-\underline{E}^{-1}(s+\rho)(\underline{A}+\rho \underline{I})\right]=g_{A+\rho I}(s+\rho) \operatorname{det}_{2}\left[\underline{I}-\underline{E}^{-1}(s+\rho)(\underline{Q}+\rho \underline{I})\right]
$$

for each $s \in \Omega$, where $g_{A+\rho I}(s+\rho)$ is analytic and vanishes nowhere on $\Omega$. By applying Lemma $2.3^{\prime}$ to the closed-loop term $\operatorname{det}_{2}\left[\underline{I}-\underline{E}^{-1}(s+\rho)\left(\underline{A}_{c}+\rho \underline{I}\right)\right]$, we readily obtain

$$
\operatorname{det}_{2}\left[\underline{I}-\underline{E}^{-1}(s+\rho)\left(\underline{A}_{c}+\rho \underline{I}\right)\right]=g_{A_{c}+\rho I}(s+\rho) \operatorname{det}_{2}\left[\underline{I}-\underline{E}^{-1}(s+\rho)\left(\underline{Q}_{c}+\rho \underline{I}\right)\right]
$$

for each $s \in \Omega$, where $g_{A_{c}+\rho I}(s+\rho)$ is analytic and vanishes nowhere on $\Omega$. Here, $\underline{Q}_{c}:=\mathcal{T}\left\{Q_{c}\right\}$ with $Q_{c}$ being the constant matrix of the Floquet factorization of the ${ }_{\text {transition matrix }} \Phi_{c}\left(t, t_{0}\right)$ of the closed-loop FDLCP system (3.1). Thus, relation (3.5) can be rewritten as

$$
\begin{aligned}
\operatorname{det}_{2}[\underline{I}+\underline{K} \underline{G}(s)] \exp \{-\Delta(s+\rho)\} \\
=\frac{g_{A_{c}+\rho I}(s+\rho) \operatorname{det}_{2}\left[\underline{I}-\underline{E}^{-1}(s+\rho)\left(\underline{Q}_{c}+\rho \underline{I}\right)\right]}{g_{A+\rho I}(s+\rho) \operatorname{det}_{2}\left[\underline{I}-\underline{E}^{-1}(s+\rho)(\underline{Q}+\rho \underline{I})\right]} .
\end{aligned}
$$

Now we concentrate the attention on the right-hand side of (3.8). By Lemma 2.4 stated in Remark 2, the right-hand side of (3.8) is just $g_{A_{c}+\rho I}(s+\rho) f_{Q_{c}+\rho I}(s+\rho) /$ $g_{A+\rho I}(s+\rho) f_{Q+\rho I}(s+\rho)=: d(s)$, which is analytic on $\Omega$ except at the zeros of $f_{Q+\rho I}(s+\rho)$ contained in $\Omega$. It is also clear that $d(s)$ is meromorphic by Theorem 15.12 of [24]. Apparently, by the definition of $\Omega$, only finitely many zeros of $f_{Q_{c}+\rho I}(s+\rho)$ and $f_{Q+\rho I}(s+\rho)$ are contained in $\Omega$, which in particular implies that (3.8) is not identically zero over $\Omega$. These facts imply that the argument principle about complex functions applies to $d(s)$ if the right-hand side of (3.8) never vanishes at each $s \in \partial \Omega\left(=N_{r}\right)$ (this guarantees that there are no zeros of $f_{Q_{c}+\rho I}(s+\rho)$ located on $\left.N_{r}\right)$. Note by Remark 2 that the sets of zeros of $f_{Q+\rho I}(s+\rho)$ and $f_{Q_{c}+\rho I}(s+\rho)$ are actually independent of $\rho$ and just the sets of the eigenvalues of the open- and closed-loop operators, $Q-\underline{E}(j 0)$ and $Q_{c}-\underline{E}(j 0)$, respectively. Furthermore, it follows readily that the right-hand side of ${ }^{c}(3.8)$ never vanishes on the segments $N_{b c}, N_{c d}$, and $N_{d a}$ of $N_{r}$ because of the assumption that they do not go through the closed-loop eigenvalues. Hence, some straightforward discussions will lead to the desired assertions.

Remark 3. It should be stressed that $n_{\text {us }}$ indicates the number of unstable eigenvalues of $\underline{Q}-\underline{E}(j 0)$ in the closed right-half portion of the fundamental strip. However, it is easy to see that $n_{\mathrm{us}}$ equals the number of the unstable eigenvalues of $Q$ in the whole closed right-half plane.

3.4. Equivalent interpretation of Theorem 3.1. In this subsection we show that Theorem 3.1 can have a more explicit expression which will provide convenience for implementing Theorem 3.1. To this end, let us return to (3.2) and compute the 
$\operatorname{det}_{2}$ of $\underline{I}+\underline{K} \underline{G}(s)$ again but after the harmonic transfer operator $\underline{G}(s)$ has been rewritten as $\underline{G}(s)=\underline{\hat{C}}(\underline{E}(s)-Q)^{-1} \underline{\hat{B}}$ by means of Lemma 2.1 and (2.7). That is, we compute

$$
\operatorname{det}_{2}[\underline{I}+\underline{K} \underline{G}(s)]=\operatorname{det}_{2}\left[\underline{I}+\underline{K} \underline{\hat{C}}(\underline{E}(s)-\underline{Q})^{-1} \underline{\hat{B}}\right] .
$$

It is not hard to see that all the arguments around (3.2)-(3.6) can be repeated on the operator $\underline{K} \underline{\hat{C}}(\underline{E}(s)-\underline{Q})^{-1} \underline{\hat{B}} \in \mathcal{C}_{2}\left(l_{2}\right)$. Then we can conclude that

$$
\operatorname{det}_{2}[\underline{I}+\underline{K} \underline{G}(s)] \exp \{-\tilde{\Delta}(s+\rho)\}=\frac{\operatorname{det}_{2}\left[\underline{I}-\underline{E}^{-1}(s+\rho)\left(\underline{\tilde{A}}_{c}+\rho \underline{I}\right)\right]}{\operatorname{det}_{2}\left[\underline{I}-\underline{E}^{-1}(s+\rho)(\underline{Q}+\rho \underline{I})\right]}
$$

with $\underline{\tilde{A}}_{c}:=\underline{Q}-\underline{\hat{B}} \underline{K} \underline{\hat{C}}$ and

$$
\tilde{\Delta}(s+\rho):=-\operatorname{tr}\left(\underline{E}^{-1}(s+\rho)(\underline{Q}+\rho \underline{I})(\underline{E}(s)-\underline{Q})^{-1} \underline{\hat{B}} \underline{K} \underline{\hat{C}}\right) .
$$

Now we observe that $\underline{E}(j 0)-\underline{\tilde{A}}_{c}=\underline{P}^{-1}\left(\underline{E}(j 0)-\underline{A}_{c}\right) \underline{P}$, where the similarity transformation formula is used. This equation clearly says that every eigenvalue of $\underline{E}(j 0)-\underline{\tilde{A}}_{c}$ is also an eigenvalue of $\underline{E}(j 0)-\underline{A}_{c}$ and vice versa. This implies that one can test stability of the closed-loop system $G_{c}$ by that of the closed-loop system $\tilde{G}_{c}:\left(\tilde{A}_{c}(t):=\right.$ $Q-\hat{B}(t) K(t) \hat{C}(t), \hat{B}(t), \hat{C}(t))$. Combining this fact with (3.10), we have the following corollary about Theorem 3.1.

Corollary 3.2. Suppose all the assumptions of Theorem 3.1 hold. Take an arbitrary positive number $\rho$ and a sufficiently small number $r$ according to the open-loop eigenvalue condition of $Q-\underline{E}(j 0)$ on the imaginary axis such that $\rho>r \geq 0$. Consider the harmonic transfer operator $\underline{G}(s)$ and the scalar function $\tilde{\Delta}(s+\rho)$ given in (3.11). Then the closed-loop system $G_{c}$ is asymptotically stable if and only if the modified Nyquist locus, $\operatorname{det}_{2}[\underline{I}+\underline{K} \underline{G}(s)] \exp \{-\tilde{\Delta}(s+\rho)\}: N_{a b} \rightarrow \mathbf{C}$ vanishes nowhere on $N_{a b}$ and encircles the origin $n_{\mathrm{us}}$ times in the counterclockwise sense.

The Nyquist locus in Theorem 3.1 is defined by $\operatorname{det}_{2}[\underline{I}+\underline{K} \underline{G}(s)] \exp \{-\Delta(s+\rho)\}$ : $N_{a b} \rightarrow \mathbf{C}$, while that of Corollary 3.2 is defined by $\operatorname{det}_{2}[\underline{I}+\underline{K} \underline{G}(s)] \exp \{-\tilde{\Delta}(s+$ $\rho)\}: N_{a b} \rightarrow$ C. That is, in Corollary 3.2 one only needs to compute $\tilde{\Delta}(s+\rho)$ instead of $\Delta(s+\rho)$. If we further compute $\operatorname{det}_{2}[\underline{I}+\underline{K} \underline{G}(s)]$ by the right-hand relation of (3.9), then the block-diagonal structures of $(\underline{E(s)}-Q)^{-1}$ and $\underline{E}^{-1}(s+\rho)(Q+$ $\rho \underline{I})(\underline{E}(s)-Q)^{-1}$ will bring us convenience in plotting the modified Nyquist locus $\operatorname{det}_{2}[\underline{I}+\underline{K} \underline{G}(s)] \exp \{-\tilde{\Delta}(s+\rho)\}: N_{a b} \rightarrow \mathbf{C}$ as we will see in the next section.

Remark 4 . Note that only the "DC-part" of $\hat{B}(t) K(t) \hat{C}(t)$ contributes to $\tilde{\Delta}(s+\rho)$. In other words, if the "DC-part" of $\hat{B}(t) K(t) \hat{C}(t)$ is zero, then the exponential part on the left-hand side of (3.10) can be dropped. As a side note, we point out that $\Delta(s+\rho)$ does not equal $\tilde{\Delta}(s+\rho)$ since $\underline{P}$ and $\underline{E}^{-1}(s)$ do not commute, and $\operatorname{det}_{2}\left[\underline{I}-\underline{E}^{-1}(s) \underline{Q}\right] \neq$ $\operatorname{det}_{2}\left[\underline{I}-\underline{E}^{-1}(s) \underline{A}\right]$ in general.

Remark 5 . Since any periodically time-varying state matrix $A(t)$ can be rewritten in the form of $A_{\text {const }}+\tilde{A}(t)$ with $A_{\text {const }}$ being a constant matrix, stability of the FDLCP system with the state matrix $A(t)$, no matter this FDLCP system itself is open- or closed-loop, can be easily tested by recasting the stability problem as a closed-loop stability problem with $\left(A_{\text {const }}, I, I\right)=(Q, I, I)$ being the open-loop system matrices and $-\tilde{A}(t)$ being (treated as) the feedback gain. This recasting technique means that Corollary 3.2 can be easily applied without computing the transition matrix of any FDLCP models. Having this recasting technique in mind, a finitedimensional truncated implementation of the modified Nyquist criterion is developed in the following section. 
4. Implementation of the 2-regularized Nyquist criterion. In the previous section, generalized Nyquist criteria were developed for asymptotic stability of FDLCP systems. Unfortunately, however, it is hard to implement them directly due to the infinite-dimensionality of the harmonic transfer operator and operators involved in the determinant and trace computations. In this section, we consider the implementation problem of the 2-regularized Nyquist criterion in Corollary 3.2 through the staircase truncations on $\underline{G}(s)$ and other infinite-dimensional operators involved, and then the closed-loop stability analysis is reduced to that of a finite-dimensional LTI continuous-time system in an asymptotic sense.

The staircase truncation is first proposed for the $H_{\infty}$ norm computation in FDLCP systems [30]. It should be noted that although the same truncation is adopted, the convergence problem in the $H_{\infty}$ norm computation and that in the Nyquist locus plotting are essentially different. More precisely, in the $H_{\infty}$ norm case convergence is related to infinite summations, while in the Nyquist locus case of this paper convergence pertains to infinite products. This discrepancy alerts us that just sketching the convergence proof might mislead the reader in understanding the implementation algorithm, and thus we keep the arguments in their complete form. Another benefit to make the convergence arguments in this way is that the inequalities in the convergence arguments can explicitly provide us with estimation formulas of the truncation size, though we will not deal with the size estimation problem in the paper due to the space limitation.

For simplicity, the discussions are given for the case of $\underline{K}=\underline{I}$ throughout this section. This brings no loss of generality if we notice that one can always treat $\underline{K} \underline{C}$ as a single operator in the harmonic return difference operator $\underline{I}+\underline{K} \underline{G}(s)$.

4.1. Truncation description. In this subsection we describe the staircase truncation. Strictly speaking, the staircase truncation is two-step: first skew truncate $\underline{G}(s)$ to $\underline{G}_{[N]}(s)$, and then truncate $\underline{G}_{[N]}(s)$ in a staircase fashion to $\underline{G}_{[N, M]}(s)$. Namely, we take an integer $N \geq 1$ and approximate $\underline{G}(s)=\underline{\hat{C}}(\underline{E}(s)-\underline{Q})^{-1} \underline{\hat{B}}$ by

$$
\underline{G}_{[N]}(s)=\underline{\hat{C}}_{[N]}(\underline{E}(s)-\underline{Q})^{-1} \underline{\hat{B}}_{[N]},
$$

where $\underline{B}_{[N]}:=\mathcal{T}\left\{\hat{B}_{N}(t)\right\}$. Here $\hat{B}_{N}(t):=\sum_{m=-N}^{N} \hat{B}_{m} e^{j m \omega_{h} t}$ with $\left\{\hat{B}_{m}\right\}$ being the Fourier coefficient sequence of $\hat{B}(t)$. Similarly, $\underline{\hat{C}}_{[N]}$ is constructed in terms of $\left\{\hat{C}_{m}\right\}$, which is the Fourier coefficient sequence of $\hat{C}(t)$. Only the skew truncation cannot reduce the $\operatorname{det}_{2}$ computation to a finite-dimensional one, and thus we introduce the staircase truncation on $\underline{G}(s)$ as follows:

$$
\underline{G}_{[N, M]}(j \varphi)=\underline{\hat{C}}_{[N, M]}\left(\underline{E}_{M}(s)-\underline{Q}_{M}\right)^{-1} \underline{\hat{B}}_{[N, M]} .
$$

Here, the infinite-dimensional matrix $\underline{B}_{[N, M]}:=\operatorname{diag}\left[\ldots, \hat{B}_{N M}, \hat{B}_{N M}, \hat{B}_{N M}, \ldots\right]$ is defined with

$$
\hat{B}_{N M}=\underbrace{\left[\begin{array}{ccccc}
\hat{B}_{0} & \cdots & \hat{B}_{-N} & & 0 \\
\vdots & \ddots & & \ddots & \\
\hat{B}_{N} & & \ddots & & \hat{B}_{-N} \\
& \ddots & & \ddots & \vdots \\
0 & & \hat{B}_{N} & \cdots & \hat{B}_{0}
\end{array}\right]}_{(2 M+1)-\text { blocks }},
$$


where we assume $M \geq N+1$. The infinite-dimensional matrix $\hat{\underline{C}}_{[N, M]}$ is defined similarly to $\underline{\hat{B}}_{[N, M]}$ but in terms of the Fourier coefficients of $\hat{C}(t)$. Furthermore, the infinite-dimensional but block-diagonal operators $\underline{E}(s)$ and $\underline{Q}$ are partitioned into diagonal blocks accordingly. That is,

$$
\begin{aligned}
\underline{Q}_{M} & :=\operatorname{diag}\left[\ldots, Q_{M}, Q_{M}, Q_{M}, \ldots\right](=\underline{Q}) \\
\underline{E}_{M}(s) & :=\operatorname{diag}\left[\ldots, E_{M,-1}(s), E_{M 0}(s), E_{M 1}(s), \ldots\right](=\underline{E}(s))
\end{aligned}
$$

with $Q_{M}=\operatorname{diag}[Q, Q, \ldots, Q]$ being $(2 M+1) \times(2 M+1)$ and

$$
E_{M m}(s)=\operatorname{diag}\left[\varphi_{m(2 M+1)-M}(s) I, \ldots, \varphi_{m(2 M+1)}(s) I, \ldots, \varphi_{m(2 M+1)+M}(s) I\right],
$$

where $m \in \mathbf{Z}$.

4.2. Truncation convergence. To state the final result, we need to establish convergence lemmas associated with the staircase truncation on the harmonic transfer operator in the $\operatorname{det}_{2}$ and trace sense. In this subsection, we show relevant convergence lemmas for the suggested truncation treatment.

LEMma 4.1. Assume in the FDLCP system (2.1) that $A(t) \in L_{\mathrm{PCD}}[0, h]$ and $B(t), C(t) \in L_{\mathrm{CAC}}[0, h]$, and the domain $\Omega$ satisfies $\mathrm{A} 1$. Then for any $\mu>0$, there exists an integer $N_{0}(\mu)>0$ such that $\left|\operatorname{det}_{2}\left[\underline{I}+\underline{G}_{[N]}(s)\right]-\operatorname{det}_{2}[\underline{I}+\underline{G}(s)]\right|<\mu(\forall N \geq$ $\left.N_{0}(\mu), \forall s \in \partial \Omega\right)$.

On the basis of Lemma 4.1, to show the convergence that $\operatorname{det}_{2}\left[\underline{I}+\underline{G}_{[N, M]}(s)\right] \rightarrow$ $\operatorname{det}_{2}[\underline{I}+\underline{G}(s)]$ as $N, M \rightarrow \infty$, it suffices to show that $\operatorname{det}_{2}\left[\underline{I}+\underline{G}_{[N, M]}(s)\right] \rightarrow \operatorname{det}_{2}[\underline{I}+$ $\left.\underline{G}_{[N]}(s)\right]$ as $M \rightarrow \infty$ for each fixed $N>0$. This is answered by the following lemma.

LEMMA 4.2. Assume in the FDLCP system (2.1) that $A(t) \in L_{\mathrm{PCD}}[0, h]$ and $B(t), C(t) \in L_{\mathrm{CAC}}[0, h]$, and the domain $\Omega$ satisfies $\mathrm{A} 1$. Then for any $\mu>0$ and fixed $N>0$, there exists an integer $M_{0}(N, \mu)>0$ such that $\mid \operatorname{det}_{2}\left[\underline{I}+\underline{G}_{[N, M]}(s)\right]-\operatorname{det}_{2}[\underline{I}+$ $\left.\underline{G}_{[N]}(s)\right] \mid<\mu\left(\forall M \geq M_{0}(N, \mu), \forall s \in \partial \Omega\right)$.

Now we apply the staircase truncation on the infinite-dimensional matrix $\underline{\hat{B}} \underline{\hat{C}}$ as we do on $\underline{G}(s)$, and get the truncated version $\widehat{\widehat{B C}}_{[N, M]}$, which is defined similarly to $\underline{\hat{B}}_{[N, M]}$ but in terms of the Fourier coefficients of $\hat{B}(t) \hat{C}(t)$. Based on this truncation, we further define

$$
\begin{aligned}
\tilde{\Delta}_{[N, M]}(s+\rho) & :=-\operatorname{tr}\left(\underline{E}^{-1}(s+\rho)(\underline{Q}+\rho \underline{I})(\underline{E}(s)-\underline{Q})^{-1} \widehat{\widehat{B C}}_{[N, M]}\right) \\
& =-\sum_{m} \operatorname{tr}\left(\tilde{\Delta}_{m[N, M]}(s+\rho)\right),
\end{aligned}
$$

where $\underline{I}_{M}$ is defined similarly to $\underline{Q}_{M}$ but in terms of the identity matrix $I$ and

$$
\tilde{\Delta}_{m[N, M]}(s+\rho):=E_{M m}^{-1}(s+\rho)\left(Q_{M}+\rho I_{M}\right)\left(E_{M m}(s)-Q_{M}\right)^{-1} \widehat{B C}_{N M}
$$

with $m \in \mathbf{Z}$ and $I_{M}$ being the $(2 M+1) \times(2 M+1)$ blockwise identity. The matrix $\widehat{B C}_{N M}$ is defined similarly to $\hat{B}_{N M}$ but in terms of the Fourier coefficients of $\hat{B}(t) \hat{C}(t)$. Then by repeating arguments similar to those in the proofs of Lemmas 4.1 and 4.2 , the following lemma can be shown.

LEMMA 4.3. Assume in the FDLCP system (2.1) that $A(t) \in L_{\mathrm{PCD}}[0, h]$ and $B(t), C(t) \in L_{\mathrm{CAC}}[0, h]$, and the domain $\Omega$ satisfies $\mathrm{A} 1$ and $\mathrm{A} 2^{\prime}$. Then for any $\mu>$ 0 and fixed $N>0$, there exist integers $N_{1}(\mu)>0$ and $M_{1}(N, \mu)>0$ such that $\left|\tilde{\Delta}_{[N, M]}(s+\rho)-\tilde{\Delta}(s+\rho)\right|<\mu\left(\forall N \geq N_{1}(\mu), M \geq M_{1}(N, \mu), \forall s \in \partial \Omega\right)$. 
Combining Lemmas 4.1, 4.2, and 4.3, we can get a tight estimation of $\operatorname{det}_{2}[\underline{I}+$ $\underline{G}(s)] \exp \{-\tilde{\Delta}(s+\rho)\}$ by $\operatorname{det}_{2}\left[\underline{I}+\underline{G}_{[N, M]}(s)\right] \exp \left\{-\tilde{\Delta}_{[N, M]}(s+\rho)\right\}$. Now we show that the latter can be reduced to finite-dimensional computations. Indeed, by definition, we have

$$
\begin{aligned}
\operatorname{det}_{2} & {\left[\underline{I}+\underline{G}_{[N, M]}(s)\right] \exp \left\{-\tilde{\Delta}_{[N, M]}(s+\rho)\right\} } \\
= & \prod_{m} \prod_{k}\left(1+\lambda_{k}\left(G_{m[N, M]}(s)\right)\right) \exp \left\{-\lambda_{k}\left(G_{m[N, M]}(s)\right)\right\} \\
& \times \exp \left\{-\sum_{m} \operatorname{tr}\left(\tilde{\Delta}_{m[N, M]}(s+\rho)\right)\right\},
\end{aligned}
$$

where the finite-dimensional matrix $G_{m[N, M]}(s)$ is given by

$$
G_{m[N, M]}(s):=\hat{C}_{N M}\left(E_{M m}(s)-Q_{M}\right)^{-1} \hat{B}_{N M} \quad(m \in \mathbf{Z}) .
$$

LEMMa 4.4. Assume in the FDLCP system (2.1) that $A(t) \in L_{\mathrm{PCD}}[0, h]$ and $B(t), C(t) \in L_{\mathrm{CAC}}[0, h]$, and the domain $\Omega$ satisfies $\mathrm{A} 1$ and $\mathrm{A} 2^{\prime}$. Then for any fixed $N$, it holds uniformly over $s \in \partial \Omega$ that

$$
\left\{\begin{array}{l}
\lim _{M \rightarrow \infty} \prod_{m \neq 0} \prod_{k}\left(1+\lambda_{k}\left(G_{m[N, M]}(s)\right)\right) \exp \left\{-\lambda_{k}\left(G_{m[N, M]}(s)\right)\right\}=1, \\
\lim _{M \rightarrow \infty} \sum_{m \neq 0} \operatorname{tr}\left(\tilde{\Delta}_{m[N, M]}(s+\rho)\right)=0 .
\end{array}\right.
$$

On the basis of Lemma 4.4 and (4.6), it follows readily that for any $\mu>0$ and fixed $N>0$, there exists an integer $M_{2}(N, \mu)$ such that $\forall M \geq M_{2}(N, \mu)$ and $\forall s \in \partial \Omega$

$$
\begin{aligned}
& \mid \operatorname{det}_{2}\left[\underline{I}+\underline{G}_{[N, M]}(s)\right] \exp \left\{-\tilde{\Delta}_{[N, M]}(s+\rho)\right\} \\
& \quad-\operatorname{det}_{2}\left[I_{M}+G_{0[N, M]}(s)\right] \exp \left\{-\operatorname{tr}\left(\tilde{\Delta}_{0[N, M]}(s+\rho)\right)\right\} \mid<\mu .
\end{aligned}
$$

A complete proof for (4.9) is given in Appendix B to keep our mainstream discussions clear.

4.3. Finite-dimensional 2-regularized Nyquist criterion. Summarizing the above discussions, we are led immediately to the following theorem, which reduces the Nyquist criterion of Corollary 3.2 to a finite-dimensional one in an asymptotic sense.

TheOrem 4.5. Suppose in the FDLCP system (2.1) that $A(t), B(t), C(t) \in$ $L_{\mathrm{CAC}}[0, h] \cap L_{\mathrm{PCD}}[0, h]$. Let $n_{\mathrm{us}}$ denote the number of the unstable eigenvalues of the open-loop Floquet state operator $\underline{Q}-\underline{E}(j 0)$ in the fundamental strip $\mathbf{C}_{F}$ defined in (3.7). Take an arbitrary positive number $\rho$ and a sufficiently small number $r$ according to the open-loop eigenvalue condition of $Q-\underline{E}(j 0)$ on the imaginary axis such that $\rho>r \geq 0$. If $N$ and $M$ are large enough truncation parameters satisfying $M \geq N+1$, then the closed-loop system $G_{c}$ is asymptotically stable if and only if the modified Nyquist locus, $\operatorname{det}_{2}\left[I_{M}+G_{0[N, M]}(s)\right] \exp \left\{-\operatorname{tr}\left(\tilde{\Delta}_{0[N, M]}(s+\rho)\right)\right\}: N_{a b} \rightarrow \mathbf{C}$, vanishes nowhere on $N_{a b}$ and encircles the origin $n_{\mathrm{us}}$ times in the counterclockwise sense. $\tilde{\Delta}_{0[N, M]}(s+\rho)$ and $G_{0[N, M]}(s)$ are defined in (4.5) and (4.7), respectively, with $m=0$.

Remark 6. Recall the recasting treatment suggested in Remark 5, by which $A_{\text {const }}$ can be taken in such a way that the "DC-part" of $\tilde{A}(t)$ is zero. It would be worth noting that $\operatorname{tr}\left(\tilde{\Delta}_{0[N, M]}(s+\rho)\right)$ will be identically zero in such a recasting treatment, and thus the exponential part can be dropped in the (modified) Nyquist locus (see Remark 4). 
5. Numeric examples. Consider asymptotic stability of the lossy Mathieu equation by means of Theorem 4.5. The lossy Mathieu equation was frequently encountered in such studies as the rolling motion of ships [1], the flapping dynamics of the helicopter rotor blade [26], and the motion of a pendulum with a periodically excited support [12]. A comprehensive study about this differential equation can be found in [23] and [25]. It turns out to be one of the most widely studied FDLCP models in the literature, and hence using the lossy Mathieu equation as our numeric example is reasonable. More precisely, the lossy Mathieu equation is given by

$$
\left.\ddot{x}(t)+2 \xi \dot{x}(t)=\left[1-2 \beta \cos \omega_{h} t\right] u(t), \quad \omega_{h}=2 \quad \text { (i.e., } h=\pi\right)
$$

which leads to the state-space model

$$
A(t)=\left[\begin{array}{cc}
0 & 1 \\
0 & -2 \xi
\end{array}\right], \quad B(t)=\left[\begin{array}{c}
0 \\
1-2 \beta \cos \omega_{h} t
\end{array}\right], \quad C(t)=\left[\begin{array}{ll}
1 & 0
\end{array}\right],
$$

where the open-loop state matrix $A(t)$ is constant but the input matrix $B(t)$ is a $\pi$-periodic time-varying matrix, each entry of which is continuous and differentiable on $[0, h]$. In other words, in the open-loop system, $Q=A(t)$ and $P(t, 0)=I$. Now we introduce the output feedback $u(t)=-k y(t)$, where $k$ is a scalar constant. This leads to a closed-loop FDLCP system with a $\pi$-periodic time-varying state matrix, and our problem is to test the closed-loop stability by Theorem 4.5.

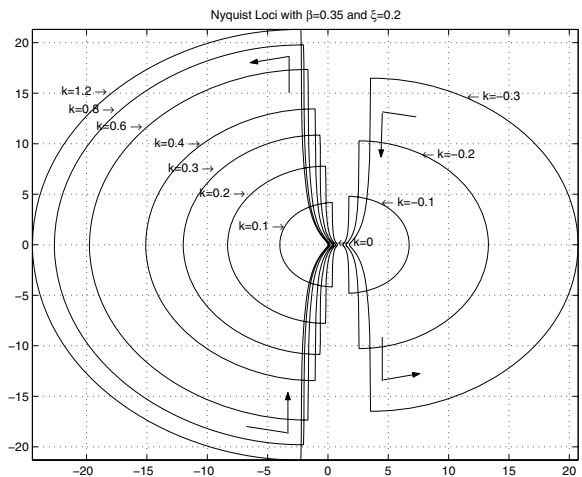

(a) Nyquist loci

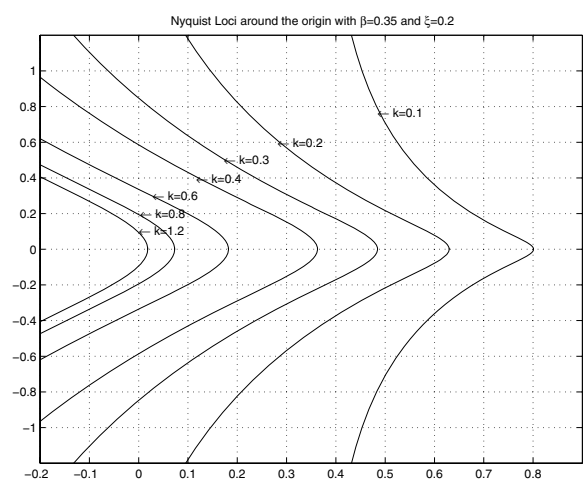

(b) Nyquist loci around the origin

FIG. 5.1. 2-Regularized Nyquist loci with the output feedback gain $k$ varying from 1.2 to -0.3 in the case of $\beta=0.35$ and $\xi=0.2$ ( $N_{r}=N_{0.05}$, the arrows indicate the Nyquist locus direction).

It is clear that the open-loop system has a zero eigenvalue. If we take a Nyquist contour as described in Figure 3.1, the corresponding region enclosed by this Nyquist contour has one unstable open-loop eigenvalue of the operator $Q-\underline{E}(j 0)$, assuming that $\xi>0$. Note also that the Fourier series expansion of $B(t)$ has nonzero terms only up to the first harmonic wave. Since $P(t, 0)=I$, it follows that the skew truncation can be dispensed with, and only the staircase truncation on the corresponding (openloop) harmonic transfer operator is enough. Figures 5.1 and 5.2 give the (modified) Nyquist loci under different parameters $\beta, \xi$ while the output feedback gain $k$ varies from 1.2 to -0.3 . In the computations, the staircase truncation parameter $M=10$, the shift factor $\rho=0.1$, and the bypassing radius $r=0.05$ are taken for simplicity. The computation results show that there are no numerically discernible differences among the Nyquist loci when larger $M$ 's are taken, for example, when $M=20$. 
From Figure 5.1, it can be asserted that in the case of $\beta=0.35$ and $\xi=0.2$, the closed-loop FDLCP system is stable when the feedback gain $k$ is relatively small, while the closed-loop system slides to the stable/unstable boundary when the feedback gain $k$ is overstrong (i.e., $k \geq 1.2$ ). However, in the case of $\beta=0.35$ and $\xi=0.5$, whose Nyquist loci are given in Figure 5.2, stability of the closed-loop FDLCP system has relatively strong robustness to the output feedback gain variation. From Figures 5.1 and 5.2, the Nyquist loci when positive feedback is applied, i.e., $k \leq 0$, tell us that the closed-loop FDLCP systems are unstable in both cases.

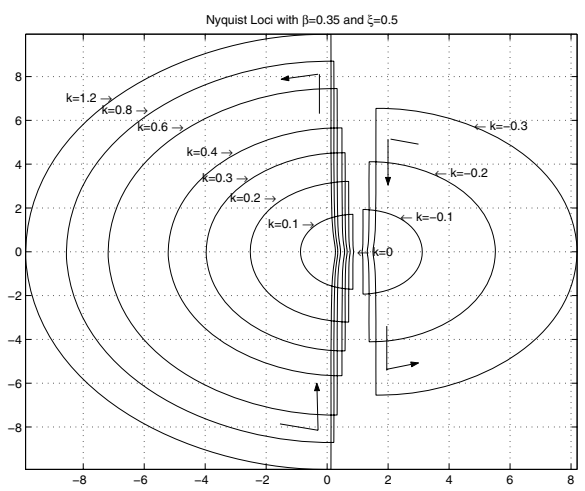

(a) Nyquist loci

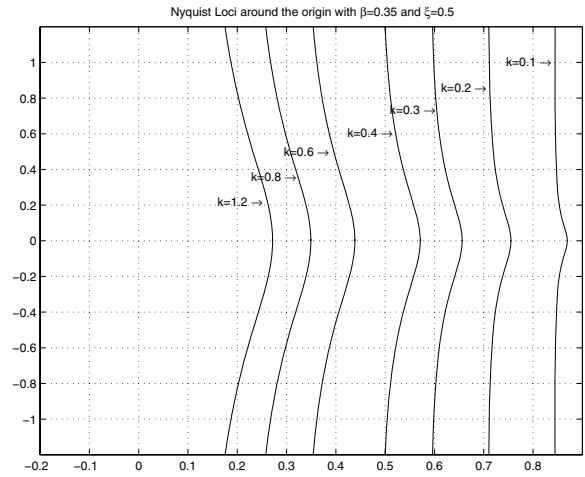

(b) Nyquist loci around the origin

FIG. 5.2. 2-Regularized Nyquist loci with the output feedback gain $k$ varying from 1.2 to -0.3 in the case of $\beta=0.35$ and $\xi=0.5\left(N_{r}=N_{0.05}\right.$, the arrows indicate the Nyquist locus direction).

Finally, we observe that Nyquist loci under different bypassing radii $r$ 's can also reveal some structural features of the open-loop FDLCP systems. For example, in the case of $\beta=0.5, \xi=0.2$, and $k=0.4$, Figure 5.3 gives the Nyquist loci with the bypassing radius being $r=0.05,0.04$, and 0.03 , respectively. One can see that as $r \rightarrow 0$, the Nyquist locus goes to infinity on the portion corresponding to the bypassing semicircle. This clearly reflects the fact that the open-loop system has an unstable eigenvalue at the origin.

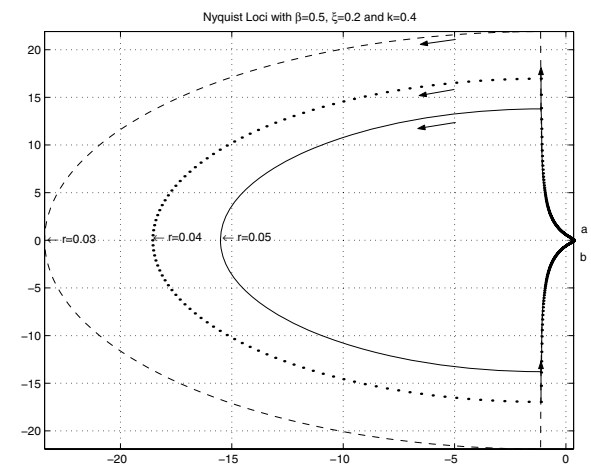

FIG. 5.3. 2-Regularized Nyquist loci with different bypassing radii $r$.

The stability results about the lossy Mathieu equation here coincide with those derived through the approximate modeling approach [31], which also gives necessary and sufficient stability conditions for FDLCP systems via the so-called harmonic 
Lyapunov equation. Note that the lossy Mathieu equation may be reexpressed by a periodic differential equation similar to that of Exercise 1.5.6 of [12]. Hence, the stability assertion for Exercise 1.5.6 of [12] confirms that stability analysis through the Nyquist criterion upon the lossy Mathieu equation is effective.

6. Conclusion. In this paper, we established a generalized Nyquist criterion in FDLCP systems by means of the 2-regularized determinant related to the openloop harmonic transfer operator. This work is inspired by the fact that the harmonic transfer operators of most practical FDLCP systems, which are defined via the Fourier series analysis of the system matrices, are Hilbert-Schmidt operators; that is, the use of the 2-regularized determinants can be validated for a large class of FDLCP systems, while the use of the usual determinant cannot. This criterion makes it possible to test the closed-loop asymptotic stability through open-loop analysis in a much more general FDLCP setting, compared to the Hill-determinant and trace class operator determinant techniques. The Hill-determinant defined on the harmonic transfer operator [26] is hard to validate in general. Moreover, by using the recasting technique suggested in Remark 5, the generalized Nyquist criterion can be applied to both open- and closed-loop FDLCP systems without involving the transition matrix and Floquet factorization computations, and thus can be implemented via finite-dimensional conditions in an asymptotic sense. In addition, it is clear that the 2-regularized Nyquist criterion applies to both SISO (single input/single output) and MIMO (multi input/multi output) cases. Observations (say by Theorem 7.4 of [11]) indicate that the Nyquist locus in the 2-regularized determinant sense is also continuous with regard to the periodically time-varying feedback gain $K(t)$, and thus can be utilized in robustness analysis. This is left as one of our subsequent research topics.

Appendix A. The function $f(n)$ of an integer $n$ is defined by

$$
f(n)= \begin{cases}1, & n=0 \\ |n|^{-1}, & n \neq 0\end{cases}
$$

Then we have $\sum_{n=N+1}^{\infty} f(n)^{2}<\frac{1}{N} \quad(N \geq 1)$ and $\sum_{n=-\infty}^{\infty} f(n)^{2}<5$.

Appendix B.

Proof of Lemma 2.2. Under the assumptions on $A(t), B(t)$, and $C(t)$, the similarity transformation formulas of Lemma 2.1 apply. Thus the harmonic transfer operator can be rewritten as

$$
\underline{G}(s)=\underline{\hat{C}}(\underline{E}(s)-\underline{Q})^{-1} \underline{\hat{B}}
$$

for all $s \in \Omega \backslash \Lambda$. Here, $\underline{\hat{B}}$ and $\underline{\hat{C}}$ are bounded on $l_{2}$ by Corollary 2.2 of [9, p. 567]. Also, it is obvious that for each fixed $s \in \Omega \backslash \Lambda$, there exists a number $K(s)>0$ such that

$$
\left\|\left(\varphi_{m}(s) I-Q\right)^{-1}\right\| \leq K(s) f(m),
$$

where $f$ is defined in Appendix A. Noting the block-diagonal structure of $(\underline{E}(s)-Q)^{-1}$, it follows that $(\underline{E}(s)-\underline{Q})^{-1}$ is compact for $s \in \Omega \backslash \Lambda$. Since $\left\|(\underline{E}(s)-Q)^{-1}\right\|_{2}^{2}=$ $\sum_{m}\left\|\left(\varphi_{m}(s)-Q\right)^{-1}\right\|_{2}^{2} \leq n \sum_{m}\left\|\left(\varphi_{m}(s)-Q\right)^{-1}\right\|^{2}$, (B.2) tells that $(\underline{E}(s)-\underline{Q})^{-1} \in$ $\mathcal{C}_{2}\left(l_{2}\right)$ for each fixed $s \in \Omega \backslash \Lambda$. Noting that $\underline{\hat{B}}$ and $\underline{\hat{C}}$ are bounded on $l_{2}$, we obtain by (B.1) that $\underline{G}(s) \in \mathcal{C}_{2}\left(l_{2}\right)$ for each $s \in \Omega \backslash \Lambda$. 
It remains to show that $\|\underline{G}(s)\|_{2}$ is uniformly bounded over $s \in \partial \Omega$. To this end, let us show that there exists a number $K>0$ independent of $s$ such that

$$
\left\|\left(\varphi_{m}(s) I-Q\right)^{-1}\right\| \leq K f(m)
$$

for all $s \in \partial \Omega$. To see this, we note that if $\|Q / z\|<1$, then

$$
\begin{aligned}
& \left\|(z I-Q)^{-1}\right\|=\frac{1}{|z|} \| I-\frac{1}{z} Q+\frac{1}{z^{2}} Q^{2}-\cdots \\
& \quad \leq \frac{1}{|z|}\left(\|I\|+\left\|\frac{1}{z} Q\right\|+\left\|\frac{1}{z^{2}} Q^{2}\right\|+\cdots\right) \leq \frac{1}{|z|-\|Q\|} .
\end{aligned}
$$

Since $\left|\varphi_{m}(s)\right|>\|Q\|$ if $|m| \omega_{h}>|\operatorname{Im}(s)|+\|Q\|$, this inequality says if $|m| \omega_{h}>|\operatorname{Im}(s)|+$ $\|Q\|$, then

$$
\left\|\left(\varphi_{m}(s) I-Q\right)^{-1}\right\| \leq\left(\left|\varphi_{m}(s)\right|-\|Q\|\right)^{-1} .
$$

Thus it is clear by (2.6) that there exists an integer $m_{0}>0$ such that (B.4) holds for all integers $m \geq m_{0}$ and $s \in \partial \Omega$. Again from (2.6), there is $K_{m_{0}}>0$ independent of $s$ such that $\left\|\left(\varphi_{m}(s) I-Q\right)^{-1}\right\| \leq K_{m_{0}} f(m)$ for all $m \geq m_{0}$ and $s \in \partial \Omega$. Furthermore, for each $s \in \partial \Omega, \varphi_{m}(s)$ is not an eigenvalue of $Q$ by A1. Hence, from (2.6) there is $K_{m_{0}}^{\prime}>0$ such that for all $|m|<m_{0}$ and $s \in \partial \Omega,\left\|\left(\varphi_{m}(s) I-Q\right)^{-1}\right\| \leq K_{m_{0}}^{\prime} f(m)$. Taking $K=\max \left\{K_{m_{0}}, K_{m_{0}}^{\prime}\right\}$, (B.3) follows for any $s \in \partial \Omega$. Thus it follows from Appendix A that for any $s \in \partial \Omega,\left\|(\underline{E}(s)-\underline{Q})^{-1}\right\|_{2} \leq\left[\sum_{m} n K^{2} f(m)^{2}\right]^{1 / 2}<K^{\prime}$ for some $K^{\prime}>0$ independent of $s \in \partial \Omega$. Finally, noting that $\underline{\hat{B}}$ and $\underline{\hat{C}}$ are bounded on $l_{2}$ and $\left\|\underline{\hat{C}}(\underline{E}(s)-\underline{Q})^{-1} \underline{\hat{B}}\right\|_{2} \leq\|\underline{\hat{C}}\|_{l_{2} / l_{2}}\|\underline{\hat{B}}\|_{l_{2} / l_{2}}\left\|(\underline{E}(s)-\underline{Q})^{-1}\right\|_{2}$, the uniform boundedness of $\left\|\underline{\hat{C}}(\underline{E}(s)-\underline{Q})^{-1} \underline{\hat{B}}\right\|_{2}$ over $\partial \Omega$ follows readily.

Proof of Lemma 2.3. By the definition of $\varphi_{m}(s)$, if we write $s=x+j y$, then we obtain

$$
\left|\varphi_{m}(s)^{-1}\right|=\frac{1}{\sqrt{x^{2}+\left(y+m \omega_{h}\right)^{2}}} \leq \begin{cases}\left(\left|y+m \omega_{h}\right|\right)^{-1} \leq K_{\varphi} f(m) & (m \neq 0), \\ \left(\sqrt{x^{2}+y^{2}}\right)^{-1} \leq K_{\varphi} f(m) \quad(m=0)\end{cases}
$$

for some $K_{\varphi}>0$ independent of $s \in \Omega$, where A1 is used for $m \neq 0$ while A2 is used for $m=0$. Furthermore, we observe from (B.5) that

$$
\left\|\underline{E}^{-1}(s)\right\|_{2} \leq\left[\sum_{m} n K_{\varphi}^{2} f(m)^{2}\right]^{1 / 2} \leq K_{E}<\infty
$$

for some $K_{E}>0$ independent of $s \in \Omega$. The inequality (B.6) says that $\underline{E}^{-1}(s) \in \mathcal{C}_{2}\left(l_{2}\right)$ for each $s \in \Omega$. Since $\underline{A}$ and $\underline{Q}$ are bounded on $l_{2}, \underline{E}^{-1}(s) \underline{A}$ and $\underline{E}^{-1}(s) \underline{Q}$ belong to $\mathcal{C}_{2}\left(l_{2}\right)$ for each $s \in \Omega$.

To see (2.11), we note by Lemma 2.1 that $\underline{E}(s)-\underline{A}=\underline{P}(\underline{E}(s)-\underline{Q}) \underline{P}^{-1}$, which implies

$$
\underline{I}-\underline{E}^{-1}(s) \underline{A}=\underline{E}^{-1}(s) \underline{P} \underline{E}(s)\left(\underline{I}-\underline{E}^{-1}(s) \underline{Q}\right) \underline{P}^{-1}
$$

on the subset $l_{E}$ of $l_{2}$. Furthermore, it is already known [29] on $l_{E}$ that

$$
\underline{\tilde{P}}=\underline{E}(s) \underline{P}-\underline{P} \underline{E}(s)
$$


with $\underline{\tilde{P}}:=\mathcal{T}\left\{\frac{d}{d t} P(t, 0)\right\}$. Since $l_{E}$ is $\underline{P}^{-1}$-invariant by Lemma 2.1, using (B.8) in (B.7) gives that on $l_{E}$

$$
\begin{aligned}
\underline{I}-\underline{E}^{-1}(s) \underline{A} & =\left(\underline{P}-\underline{E}^{-1}(s) \underline{\tilde{P}}\right)\left(\underline{I}-\underline{E}^{-1}(s) \underline{Q}\right) \underline{P}^{-1} \\
& =\left(\underline{I}-\underline{E}^{-1}(s) \underline{\tilde{P}} \underline{P}^{-1}\right)\left(\underline{I}-\underline{P} \underline{E}^{-1}(s) \underline{Q} \underline{P}^{-1}\right) .
\end{aligned}
$$

Noting that all operators in (B.9) are bounded and $l_{E}$ is dense in $l_{2},(B .9)$ is true on the whole $l_{2}$. On the other hand, it is straightforward to see that $\underline{E}^{-1}(s) \underline{\tilde{P}} \underline{P}^{-1}$ and $\underline{P} \underline{E}^{-1}(s) Q \underline{P}^{-1}$ belong to $\mathcal{C}_{2}\left(l_{2}\right)$ since $\underline{E}^{-1}(s)$ does. These observations validate the following derivations:

$$
\begin{aligned}
\operatorname{det}_{2}\left[\underline{I}-\underline{E}^{-1}(s) \underline{A}\right]= & \operatorname{det}_{2}\left[\underline{I}-\underline{E}^{-1}(s) \underline{\tilde{P}} \underline{P}^{-1}\right] \operatorname{det}_{2}\left[\underline{I}-\underline{P} \underline{E}^{-1}(s) \underline{Q} \underline{P}^{-1}\right] \\
& \times \exp \left\{-\operatorname{tr}\left(\underline{E}^{-1}(s) \underline{\tilde{P}} \underline{P}^{-1} \underline{P} \underline{E}^{-1}(s) \underline{Q} \underline{P} \underline{P}^{-1}\right)\right\} \\
= & \operatorname{det}_{2}\left[\underline{I}-\underline{E}^{-1}(s) \underline{\tilde{P}} \underline{P}^{-1}\right] \operatorname{det}_{2}\left[\underline{I}-\underline{E}^{-1}(s) \underline{Q}\right] \\
& \times \exp \left\{-\operatorname{tr}\left(\underline{E}^{-1}(s) \underline{\tilde{P}} \underline{E}^{-1}(s) \underline{Q} \underline{P}^{-1}\right)\right\} \\
= & g_{A}(s) \operatorname{det}_{2}\left[\underline{I}-\underline{E}^{-1}(s) \underline{Q}\right],
\end{aligned}
$$

where $g_{A}(s):=\operatorname{det}_{2}\left[\underline{I}-\underline{E}^{-1}(s) \underline{\tilde{P}} \underline{P}^{-1}\right] \exp \left\{-\operatorname{tr}\left(\underline{E}^{-1}(s) \underline{\tilde{P}} \underline{E}^{-1}(s) Q \underline{P}^{-1}\right)\right\}$. Hence, the assertions regarding (2.11) will follow if it is shown that $g_{A}(s)$ does not vanish and is analytic over $\Omega$.

We complete the task in two steps by showing that the two components of $g_{A}(s)$ vanish nowhere on $\Omega$ and are analytic in $s$ over $\Omega$.

Step 1. It is shown that $\operatorname{det}_{2}\left[\underline{I}-\underline{E}^{-1}(s) \underline{\tilde{P}} \underline{P}^{-1}\right]$ vanishes nowhere on $\Omega$ and is analytic in $s$ over $\Omega$. To see this, we note from (B.8) that on $l_{E}$,

$$
\underline{I}-\underline{E}^{-1}(s) \underline{\tilde{P}} \underline{P}^{-1}=\underline{E}^{-1}(s) \underline{P} \underline{E}(s) \underline{P}^{-1},
$$

which says that $\underline{I}-\underline{E}^{-1}(s) \underline{\tilde{P}} \underline{P}^{-1}$ is invertible on $l_{E}$ for each $s \in \Omega$ since $\underline{E}(s)$ is invertible on $l_{E}$, and $l_{E}$ is $\underline{P}$ - and $\underline{P}^{-1}$-invariant (see Lemma 2.1). Since $l_{E}$ is dense in $l_{2}$, this, in particular, implies that $\underline{I}-\underline{E}^{-1}(s) \underline{\tilde{P}} \underline{P}^{-1}$ has a dense range in $l_{2}$. Furthermore, one can claim that $\underline{I}-\underline{E}^{-1}(s) \underline{\tilde{P}} \underline{P}^{-1}$ is one-to-one on $l_{2}$ since $\left(\underline{I}-\underline{E}^{-1}(s) \underline{\tilde{P}} \underline{P}^{-1}\right) \underline{x}=0$ implies that $\underline{x} \in l_{E}$, and thus $\underline{x}=0$ by the invertibility of $\underline{I}-\underline{E}^{-1}(s) \underline{\tilde{P}} \underline{P}^{-1}$ on $l_{E}$. On the basis of these facts, Theorem 2.7 .6 of $[21$, p. 30] tells us that the operator $\underline{I}-\underline{E}^{-1}(s) \underline{\tilde{P}} \underline{P}^{-1}$ is actually invertible on the whole $l_{2}$. This, together with the invertibility of $\underline{I}-\underline{E}^{-1}(s) \underline{Q}$, implies by (B.9) that $\underline{I}-\underline{E}^{-1}(s) \underline{A}$ is invertible on $l_{2}$. On the other hand, Property $1.8(\mathrm{e})$ of $[5, \mathrm{p} .17]$ ensures that $\operatorname{det}_{2}\left[\underline{I}-\underline{E}^{-1}(s) \underline{\tilde{P}} \underline{P}^{-1}\right] \neq 0$ on $\Omega$.

To show that $\operatorname{det}_{2}\left[\underline{I}-\underline{E}^{-1}(s) \underline{\tilde{P}} \underline{P}^{-1}\right]$ is analytic in $s$ over $\Omega$, we need some preparations. To this end, let us approximate $\underline{\tilde{P}}$ and $\underline{P}^{-1}$ by $[\underline{\tilde{\tilde{P}}}]_{N}$ and $\left[\underline{P}^{-1}\right]_{N}$, respectively, as follows:

$$
[\underline{\tilde{P}}]_{N}:=\mathcal{T}\left\{\sum_{|m| \leq N} \tilde{P}_{m} e^{j m \omega_{h} t}\right\}, \quad\left[\underline{P}^{-1}\right]_{N}:=\mathcal{T}\left\{\sum_{|m| \leq N} \check{P}_{m} e^{j m \omega_{h} t}\right\} .
$$

Here $\left\{\tilde{P}_{m}\right\}$ and $\left\{\check{P}_{m}\right\}$ are the Fourier coefficients sequences of $\tilde{P}(t, 0)$ and $P^{-1}(t, 0)$, respectively. Now let us define the operators

$$
\underline{K}(s):=-\underline{E}^{-1}(s) \underline{\tilde{P}} \underline{P}^{-1}, \quad \underline{K}_{N}(s):=-\underline{E}^{-1}(s)[\underline{\tilde{P}}]_{N}\left[\underline{P}^{-1}\right]_{N} .
$$


Since $\underline{E}^{-1}(s) \in \mathcal{C}_{2}\left(l_{2}\right)$, it follows that $\underline{K}_{N}(s) \in \mathcal{C}_{2}\left(l_{2}\right)$ for each $N$ and $s \in \Omega$. By the structure of the operators $\underline{\tilde{P}}$ and $\underline{P}^{-1}$ and (B.6), Proposition 1.3 of [9, p. 98] tells us that

$$
\begin{aligned}
\left\|\underline{K}_{N}(s)\right\|_{2} & \leq\left\|\underline{E}^{-1}(s)\right\|_{2}\left\|[\underline{\tilde{P}}]_{N}\right\|_{l_{2} / l_{2}}\left\|\left[\underline{P}^{-1}\right]_{N}\right\|_{l_{2} / l_{2}} \leq K_{E} \sum_{|m| \leq N}\left\|\tilde{P}_{m}\right\| \sum_{|m| \leq N}\left\|\check{P}_{m}\right\| \\
& \leq K_{E} \sum_{m=-\infty}^{+\infty}\left\|\tilde{P}_{m}\right\| \sum_{m=-\infty}^{+\infty}\left\|\check{P}_{m}\right\| \leq K_{2}<\infty
\end{aligned}
$$

for some $K_{1}>0$ and $K_{2}>0$ independent of $s \in \Omega$ and $N$. This is because $\tilde{P}(t, 0)$ and $P(t, 0)$ belong to $L_{\mathrm{CAC}}[0, h]$ under the assumption about $A(t)$ [29], and thus $\sum_{m=-\infty}^{+\infty}\left\|\tilde{P}_{m}\right\|<\infty$ and $\sum_{m=-\infty}^{+\infty}\left\|\check{P}_{m}\right\|<\infty$. By A2 and the form of $\underline{E}(s), \underline{K}_{N}(s)$ is analytic in $s$ over $\Omega$ in the elementwise sense since each entry of $\underline{K}_{N}(s)$ is a finite sum due to the skew-strip structure of $[\underline{\tilde{P}}]_{N}$ and $\left[\underline{P}^{-1}\right]_{N}$. In the following, we say that $\underline{K}_{N}(s)$ is an analytic $\mathcal{C}_{2}\left(l_{2}\right)$-valued function in this sense.

Now we show that $\underline{K}(s)-\underline{K}_{N}(s) \rightarrow 0$ in the norm of $\mathcal{C}_{2}\left(l_{2}\right)$ uniformly over $\Omega$ as $N \rightarrow \infty$. To see this, we note that

$$
\begin{aligned}
& \left\|\underline{K}(s)-\underline{K}_{N}(s)\right\|_{2} \leq\left\|\underline{E}^{-1}(s)\right\|_{2}\left\|\underline{\tilde{P}} \underline{P}^{-1}-[\underline{\tilde{P}}]_{N}\left[\underline{P}^{-1}\right]_{N}\right\|_{l_{2} / l_{2}} \\
& \quad \leq K_{E}\left(\left\|\underline{\tilde{P}}-[\underline{\tilde{P}}]_{N}\right\|_{l_{2} / l_{2}}\left\|\underline{P}^{-1}\right\|_{l_{2} / l_{2}}+\left\|[\underline{\tilde{P}}]_{N}\right\|_{l_{2} / l_{2}}\left\|\underline{P}^{-1}-\left[\underline{P}^{-1}\right]_{N}\right\|_{l_{2} / l_{2}}\right) .
\end{aligned}
$$

On the other hand, by the structures of $\underline{\tilde{P}}-[\underline{\tilde{P}}]_{N}$ and $\underline{P}^{-1}-\left[\underline{P}^{-1}\right]_{N}$ and the facts that $\tilde{P}(t, 0)$ and $P^{-1}(t, 0)$ belong to $L_{\mathrm{CAC}}[0, h]$, it follows readily that $\left\|\underline{\tilde{P}}-[\underline{\tilde{P}}]_{N}\right\|_{l_{2} / l_{2}}$ and $\left\|\underline{P}^{-1}-\left[\underline{P}^{-1}\right]_{N}\right\|_{l_{2} / l_{2}}$ go to zero as $N \rightarrow \infty$. These facts, together with the fact that there is an upper bound for $\left\|[\underline{\tilde{P}}]_{N}\right\|_{l_{2} / l_{2}}$ independent of $N$, imply that $\| \underline{K}(s)-$ $\underline{K}_{N}(s) \|_{2} \rightarrow 0$ uniformly over $\Omega$ as $N \rightarrow \infty$. This ensures that $\underline{K}(s)$ is an analytic $\mathcal{C}_{2}\left(l_{2}\right)$-valued function, which leads us to the desired consequence.

Step 2. It is shown that $\exp \left\{-\operatorname{tr}\left(\underline{E}^{-1}(s) \underline{\tilde{P}} \underline{E}^{-1}(s) Q \underline{P}^{-1}\right)\right\}$ does not vanish and is analytic over $s \in \Omega$. By Property 1.3(c) of [5, p. 14] and Theorem 2.1 of [9, p. 111], we have

$$
\begin{aligned}
& \left|\operatorname{tr}\left(\underline{E}^{-1}(s) \underline{\tilde{P}} \underline{E}^{-1}(s) \underline{Q} \underline{P}^{-1}\right)\right| \leq\left\|\underline{E}^{-1}(s) \underline{\tilde{P}} \underline{E}^{-1}(s) \underline{Q} \underline{P}^{-1}\right\|_{1} \\
& \quad \leq\left\|\underline{E}^{-1}(s)\right\|_{2}^{2}\|\underline{\tilde{P}}\|_{l_{2} / l_{2}}\left\|\underline{Q} \underline{P}^{-1}\right\|_{l_{2} / l_{2}} \leq K_{E}^{2}\|\underline{\tilde{P}}\|_{l_{2} / l_{2}}\left\|\underline{Q} \underline{P}^{-1}\right\|_{l_{2} / l_{2}} \leq K_{3}<\infty
\end{aligned}
$$

for some $K_{3}>0$ independent of $s \in \Omega$ since $\underline{\tilde{P}}, Q$, and $\underline{P}^{-1}$ are bounded on $l_{2}$. This inequality says clearly that $\exp \left\{-\operatorname{tr}\left(\underline{E}^{-1}(s) \underline{\tilde{P}} \underline{E}^{-1}(s) \underline{Q} \underline{P}^{-1}\right)\right\}$ does not vanish on $\Omega$.

To show that $\exp \left\{-\operatorname{tr}\left(\underline{E}^{-1}(s) \underline{\tilde{P}} \underline{E}^{-1}(s) \underline{Q} \underline{P}^{-1}\right)\right\}$ is analytic over $s \in \Omega$, by Remark 10.3 of [24, p. 197], it is enough to show that $\operatorname{tr}\left(\underline{E}^{-1}(s) \underline{\tilde{P}} \underline{E}^{-1}(s) Q \underline{P}^{-1}\right)=: t_{A}(s)$ is analytic over $s \in \Omega$. To this end, we further define the trace function

$$
\operatorname{tr}\left(\underline{E}^{-1}(s)[\underline{\tilde{P}}]_{N} \underline{E}^{-1}(s) \underline{Q}\left[\underline{P}^{-1}\right]_{N}\right)=: t_{N}(s) .
$$

It should be pointed out that for each fixed $N, \underline{E}^{-1}(s)[\underline{\tilde{P}}]_{N} \underline{E}^{-1}(s) \underline{Q}\left[\underline{P}^{-1}\right]_{N}$ belongs to $\mathcal{C}_{1}\left(l_{2}\right)$ since $\underline{E}^{-1}(s) \in \mathcal{C}_{2}\left(l_{2}\right)$. Hence, $t_{N}(s)$ is well defined. Now we observe that

$$
\left|t_{A}(s)-t_{N}(s)\right| \leq\left|\operatorname{tr}\left(\underline{E}^{-1}(s)\left(\underline{\tilde{P}}-[\underline{\tilde{P}}]_{N}\right) \underline{E}^{-1}(s) \underline{Q} \underline{P}^{-1}\right)\right|
$$




$$
\begin{aligned}
& +\left|\operatorname{tr}\left(\underline{E}^{-1}(s)[\underline{\tilde{P}}]_{N} \underline{E}^{-1}(s) \underline{Q}\left(\underline{P}^{-1}-\left[\underline{P}^{-1}\right]_{N}\right)\right)\right| \\
\leq & \left\|\left(\underline{E}^{-1}(s)\left(\underline{\tilde{P}}-[\underline{\tilde{P}}]_{N}\right) \underline{E}^{-1}(s) \underline{Q} \underline{P}^{-1}\right)\right\|_{1} \\
& +\left\|\underline{E}^{-1}(s)[\underline{\tilde{P}}]_{N} \underline{E^{-1}}(s) \underline{Q}\left(\underline{P}^{-1}-\left[\underline{P}^{-1}\right]_{N}\right)\right\|_{1} \\
\leq & \left\|\underline{E}^{-1}(s)\right\|_{2}^{2}\left\|\underline{\tilde{P}}-[\underline{\tilde{P}}]_{N}\right\|_{l_{2} / l_{2}}\left\|\underline{Q} \underline{P}^{-1}\right\|_{l_{2} / l_{2}} \\
& +\left\|\underline{E}^{-1}(s)\right\|_{2}^{2}\left\|[\underline{\tilde{P}}]_{N}\right\|_{l_{2} / l_{2}}\|\underline{Q}\|_{l_{2} / l_{2}}\left\|\underline{P}^{-1}-\left[\underline{P}^{-1}\right]_{N}\right\|_{l_{2} / l_{2}} .
\end{aligned}
$$

As seen in Step 1 that $\tilde{P}(t, 0)$ and $P^{-1}(t, 0)$ belong to $L_{\mathrm{CAC}}[0, h]$, it follows that $\left\|\underline{\tilde{P}}-[\underline{\tilde{P}}]_{N}\right\|_{l_{2} / l_{2}}$ and $\left\|\underline{P}^{-1}-\left[\underline{P}^{-1}\right]_{N}\right\|_{l_{2} / l_{2}}$ go to zero as $N \rightarrow \infty$. These facts, together with $\left\|\underline{E}^{-1}(s)\right\|_{2}^{2} \leq K_{E}^{2}$ and the fact that $\left\|[\underline{\tilde{P}}]_{N}\right\|_{l_{2} / l_{2}}$ has an upper bound independent of $N$, imply that $\left|t_{N}(s)-t_{A}(s)\right| \rightarrow 0$ as $N \rightarrow \infty$ uniformly over $s \in \Omega$. This indicates that to complete the proof, it suffices to show that for each fixed $N$, the function $t_{N}(s)$ is analytic over $s \in \Omega$.

It is easy to see from the structure of $[\underline{\tilde{P}}]_{N}$ and $\left[\underline{P}^{-1}\right]_{N}$ that the blockwise $(m, m)$ th entry on the diagonal of the operator $\underline{E}^{-1}(s)[\underline{\tilde{P}}]_{N} \underline{E}^{-1}(s) \underline{Q}\left[\underline{P}^{-1}\right]_{N}$ is

$$
\varphi_{m}^{-1}(s)\left[\tilde{P}_{N}, \ldots, \tilde{P}_{-N}\right] \operatorname{diag}\left[\varphi_{m-N}^{-1}(s), \ldots, \varphi_{m+N}^{-1}(s)\right]\left[\begin{array}{c}
Q \check{P}_{-N} \\
\vdots \\
Q \check{P}_{N}
\end{array}\right]
$$

whose trace is denoted by $t_{N m}(s)$. Obviously, $t_{N}(s)=\sum_{m=-\infty}^{\infty} t_{N m}(s)$. It is straightforward to see that $t_{N m}(s)$ is analytic over $\Omega$, and

$$
\left|t_{N m}(s)\right| \leq n K_{N}\left|\varphi_{m}^{-1}(s)\right| \max \left\{\left|\varphi_{m-N}^{-1}(s)\right|, \ldots,\left|\varphi_{m+N}^{-1}(s)\right|\right\},
$$

where $K_{N}=\left\|\left[\tilde{P}_{N}, \ldots, \tilde{P}_{-N}\right]\right\| \ldots\left\|\left[\check{P}_{-N}^{T} Q^{T}, \ldots, \check{P}_{N}^{T} Q^{T}\right]\right\|$. Furthermore, by Appendix A and (B.5),

$$
\begin{aligned}
& \left|t_{N}(s)-\sum_{|m| \leq M} t_{N m}(s)\right| \leq \sum_{|m|>M}\left|t_{N m}(s)\right| \\
& \leq n K_{N}\left[\sum_{|m|>M}\left|\varphi_{m}^{-1}(s)\right|^{2}\right]^{1 / 2}\left[\sum_{|m|>M}\left(\max \left\{\left|\varphi_{m-N}^{-1}(s)\right|, \ldots,\left|\varphi_{m+N}^{-1}(s)\right|\right\}\right)^{2}\right]^{1 / 2} \\
& \leq n K_{N}\left[\sum_{|m|>M} K_{\varphi}^{2} f^{2}(m)\right]^{1 / 2}\left[\sum_{m=-\infty}^{\infty}\left(\max \left\{\left|\varphi_{m-N}^{-1}(s)\right|, \ldots,\left|\varphi_{m+N}^{-1}(s)\right|\right\}\right)^{2}\right]^{1 / 2} \\
& \quad<n K_{N}\left[\frac{2 K_{\varphi}^{2}}{M}\right]^{1 / 2}\left[(2 N+1) K_{\varphi}^{2} f(0)^{2}+\sum_{|m| \geq 1} K_{\varphi}^{2} f^{2}(m)\right]^{1 / 2} \\
& \quad<n K_{N} K_{\varphi}^{2}\left[\frac{2}{M}\right]^{1 / 2}[(2 N+1)+5]^{1 / 2}=: n K_{N}^{\prime}\left[\frac{2}{M}\right]^{1 / 2} \rightarrow 0 \quad(M \rightarrow \infty)
\end{aligned}
$$

since $K_{N}^{\prime}<\infty$ for any fixed $N \geq 1$. The above arguments say that $\sum_{|m| \leq M} t_{N m}(s)$ converges to $t_{N}(s)$ uniformly over $s \in \Omega$. Therefore, it follows that $t_{N}(s)$ is also analytic over $s \in \Omega$.

Finally, let us show the assertion that $\left(\underline{I}-\underline{E}^{-1}(s) \underline{A}\right)^{-1}$ is bounded on $l_{2}$. To this end, we note by (B.8) that $\underline{E}^{-1}(s) \underline{P^{-1}} \underline{E}(s)=\underline{P}^{-1}+\underline{E}^{-1}(s) \underline{P}^{-1} \underline{\tilde{P}} \underline{P}^{-1}$ on $l_{E}$. Hence, $\underline{E}^{-1}(s) \underline{P}^{-1} \underline{E}(s)$ is bounded on $l_{E}$. Obviously, this implies that $\underline{P} \underline{E}^{-1}(s) \underline{P} \underline{P}^{-1} \underline{E}(s)$, 
which is the inverse of $\underline{I}-\underline{E}^{-1}(s) \underline{\tilde{P}} \underline{P}^{-1}$ by (B.11), is bounded on $l_{E}$. Since $l_{E}$ is dense in $l_{2}$, it follows that $\left(\underline{I}-\underline{E}^{-1}(s) \underline{\tilde{P}} \underline{P}^{-1}\right)^{-1}$ is also bounded on $l_{2}$. On the other hand, by the block-diagonal structure of $\left(\underline{I}-\underline{E}^{-1}(s) Q\right)^{-1}$ and the assumption about $\Omega$, it is straightforward to show that $\left(\underline{I}-\underline{E}^{-1}(s) \underline{Q}\right)^{-1}$ is also bounded on $l_{2}$. Summarizing these facts leads to the desired assertion by (B.9).

Proof of Lemma 2.4. The second equality of (2.12) follows from the 2-regularized determinant definition and the fact that $\underline{E}^{-1}(s) \underline{Q}$ has an eigenvalue at each point $\lambda_{k}(Q) /\left(s+j m \omega_{h}\right), m \in \mathbf{Z}$.

To see the assertions about $f_{Q}(s)$, we consider only the case of $n=1$ without loss of generality. The arguments are given by means of the results in Chapter 15 of [24, pp. 298-303]. More precisely, let us define the function sequence $\left\{f_{m}(s)\right\}$ by

$$
f_{m}(s):=\left(1-\frac{\lambda(Q)}{s+j m \omega_{h}}\right) \exp \left\{\frac{\lambda(Q)}{s+j m \omega_{h}}\right\} \quad(m \in \mathbf{Z}) .
$$

By A2, the function $f_{m}(s)$ is analytic on $\Omega$ and has a zero at $\lambda(Q)-j m \omega_{h}$. Note by (B.5) that $\left|\frac{\lambda(Q)}{s+j m \omega_{h}}\right| \leq|\lambda(Q)| K_{\varphi} f(m)$ for any $m \in \mathbf{Z}$ and $s \in \Omega$. Hence, there exists a finite integer $m_{0}>1$ such that $\left|\frac{\lambda(Q)}{s+j m \omega_{h}}\right| \leq 1 \forall|m| \geq m_{0}$ and $\forall s \in \Omega$, which implies by Lemma 15.8 of $[24$, p. 301] that

$$
\left|1-f_{m}(s)\right| \leq\left|\frac{\lambda(Q)}{s+j m \omega_{h}}\right|^{2} \leq|\lambda(Q)|^{2} K_{\varphi}^{2} f^{2}(m) \quad\left(\forall|m| \geq m_{0} \quad \forall s \in \Omega\right) .
$$

The above arguments tell us that

$$
\sum_{|m| \geq m_{0}}\left|1-f_{m}(s)\right| \leq \sum_{|m| \geq m_{0}}|\lambda(Q)|^{2} K_{\varphi}^{2} f^{2}(m) \leq \frac{2}{m_{0}-1} K_{\varphi}^{2}|\lambda(Q)|^{2}
$$

by Appendix A, which implies that $\sum_{m}\left|1-f_{m}(s)\right|$ is uniformly convergent on $\Omega$. Then it follows by the first conclusion of Theorem 15.6 of $\left[24\right.$, p. 300] that $\prod_{m} f_{m}(s)$ converges uniformly on $\Omega$, and thus the product $\prod_{m} f_{m}(s)$ is analytic on $\Omega$. The zeros property is a direct result of the second conclusion of Theorem 15.6 of [24, p. 300].

Proof of Lemma 4.1. First let us show that $\operatorname{det}_{2}\left[\underline{I}+\underline{G}_{[N]}(s)\right]$ is well defined for each $N$. That is, we must show that $\underline{G}_{[N]}(s) \in \mathcal{C}_{2}\left(l_{2}\right)$ for each $N$ and $s \in \partial \Omega$. To see this, we note by the assumptions about the system matrices that $\hat{B}(t)$ and $\hat{C}(t)$ belong to $L_{\mathrm{CAC}}[0, h][29]$. Furthermore, by the structures of the operators $\underline{\hat{B}}_{[N]}$ and $\hat{\underline{C}}_{[N]}$, it follows that

$$
\left\{\begin{array}{l}
\left\|\underline{\hat{B}}_{[N]}\right\|_{l_{2} / l_{2}} \leq \sum_{|m| \leq N}\left\|\hat{B}_{m}\right\| \leq \sum_{m=-\infty}^{+\infty}\left\|\hat{B}_{m}\right\| \leq K_{B}<\infty, \\
\left\|\hat{\hat{C}}_{[N]}\right\|_{l_{2} / l_{2}} \leq K_{C}<\infty
\end{array}\right.
$$

for some $K_{B}>0$ and $K_{C}>0$ that are independent of $N$. These facts, together with the definition of $\underline{G}_{[N]}(s)$ and (B.3), imply that for any $s \in \partial \Omega$

$$
\begin{aligned}
\left\|\underline{G}_{[N]}(s)\right\|_{2} & \leq\left\|\underline{\hat{B}}_{[N]}\right\|_{l_{2} / l_{2}}\left\|\hat{\hat{C}}_{[N]}\right\|_{l_{2} / l_{2}}\left\|(\underline{E}(s)-\underline{Q})^{-1}\right\|_{2} \\
& \leq K_{B} K_{C}\left[n \sum_{m} K^{2} f^{2}(m)\right]^{1 / 2}<\sqrt{5 n} K_{B} K_{C} K<\infty .
\end{aligned}
$$


Equation (B.17) gives that $\underline{G}_{[N]}(s)$ belongs to $\mathcal{C}_{2}\left(l_{2}\right)$ and $\left\|\underline{G}_{[N]}(s)\right\|_{2}$ has a uniform upper bound for all $N$ and $s \in \partial \Omega$. Similarly we can show that $\underline{G}_{[N, M]}(s) \in \mathcal{C}_{2}\left(l_{2}\right)$ and $\left\|\underline{G}_{[N, M]}(s)\right\|_{2}$ has a uniform upper bound for all $N, M$, and $s \in \partial \Omega$.

Now we show the main assertion. It is known from Theorem 7.4 of [11, p. 69] that

$$
\begin{aligned}
& \left|\operatorname{det}_{2}[\underline{I}+\underline{G}(s)]-\operatorname{det}_{2}\left[\underline{I}+\underline{G}_{[N]}(s)\right]\right| \\
& \quad \leq\left\|\underline{G}(s)-\underline{G}_{[N]}(s)\right\|_{2} \exp \left\{\frac{1}{2}\left(\|\underline{G}(s)\|_{2}+\left\|\underline{G}_{[N]}(s)\right\|_{2}+1\right)^{2}\right\} .
\end{aligned}
$$

Hence, the uniform boundedness of $\left\|\underline{G}_{[N]}(s)\right\|_{2}$ over $N$ and $s \in \partial \Omega$ together with that of $\|\underline{G}(s)\|_{2}$ over $s \in \partial \Omega$ says that to show the main convergence, it suffices to show that $\left\|\underline{G}(s)-\underline{G}_{[N]}(s)\right\|_{2} \rightarrow 0$ uniformly for all $s \in \partial \Omega$ as $N \rightarrow \infty$. To see this, we observe that

$$
\begin{aligned}
\left\|\underline{G}(s)-\underline{G}_{[N]}(s)\right\|_{2} \leq & \left\|\underline{\hat{C}}-\hat{\hat{C}}_{[N]}\right\|_{l_{2} / l_{2}}\left\|(\underline{E}(s)-\underline{Q})^{-1}\right\|_{2}\|\underline{\hat{B}}\|_{l_{2} / l_{2}} \\
& +\left\|\underline{\hat{C}}_{[N]}\right\|_{l_{2} / l_{2}}\left\|(\underline{E}(s)-\underline{Q})^{-1}\right\|_{2}\left\|\underline{\hat{B}}-\underline{\hat{B}}_{[N]}\right\|_{l_{2} / l_{2}} \\
< & \sqrt{5 n} K K_{B}\left\|\underline{\hat{C}}-\underline{\hat{C}}_{[N]}\right\|_{l_{2} / l_{2}}+\sqrt{5 n} K K_{C}\left\|\underline{\hat{B}}-\underline{\hat{B}}_{[N]}\right\|_{l_{2} / l_{2}},
\end{aligned}
$$

where (B.16) is used. Furthermore, by the skew structure of $\underline{\hat{C}}-\underline{\hat{C}}_{[N]}$, it follows that $\left\|\underline{\hat{C}}-\underline{\hat{C}}_{[N]}\right\|_{l_{2} / l_{2}} \leq \sum_{|m| \geq N}\left\|\hat{C}_{m}\right\| \rightarrow 0$ as $(N \rightarrow \infty)$ since $\hat{C}(t) \in L_{\mathrm{CAC}}[0, h]$. Similarly, by $\hat{B}(t) \in L_{\mathrm{CAC}}[0, h]$ we have $\left\|\underline{\hat{B}}-\underline{\hat{B}}_{[N]}\right\|_{l_{2} / l_{2}} \rightarrow 0$ as $N \rightarrow \infty$. Using these facts in (B.19), the desired convergence follows.

Proof of Lemma 4.2. From the proof of Lemma 4.1, we have $\underline{G}_{[N]}(s), \underline{G}_{[N, M]}(s) \in$ $\mathcal{C}_{2}\left(l_{2}\right)$ for all $N, M$, and $s \in \partial \Omega$, and $\left\|\underline{G}_{[N]}(s)\right\|_{2}$ and $\left\|\underline{G}_{[N, M]}(s)\right\|_{2}$ are uniformly bounded from above over $N, M$, and $s \in \partial \Omega$. Therefore, an inequality similar to (B.18) between $\underline{G}_{[N]}(s)$ and $\underline{G}_{[N, M]}(s)$ can be claimed. This means that to show the result, it suffices to show that

$$
\left\|\underline{G}_{[N, M]}(s)-\underline{G}_{[N]}(s)\right\|_{2} \rightarrow 0 \quad \forall s \in \partial \Omega \quad(M \rightarrow \infty)
$$

uniformly for each fixed $N>0$. To this end, we focus the attention on the inequality

$$
\begin{aligned}
\left\|\underline{G}_{[N, M]}(s)-\underline{G}_{[N]}(s)\right\|_{2} \leq & \left\|\underline{C}_{[N, M]}(\underline{E}(s)-\underline{Q})^{-1}\right\|_{2}\left\|\underline{\hat{B}}_{[N, M]}\right\|_{l_{2} / l_{2}} \\
& +\left\|\underline{\hat{C}}_{[N]}\right\|_{l_{2} / l_{2}}\left\|(\underline{E}(s)-\underline{Q})^{-1} \underline{\check{B}}_{[N, M]}\right\|_{2},
\end{aligned}
$$

where $\underline{\underline{B}}_{[N, M]}:=\underline{\hat{B}}_{[N]}-\underline{\hat{B}}_{[N, M]}$. More explicitly, it is given by the infinite-dimensional matrix

$$
\underline{\underline{B}}_{[N, M]}:=\left[\begin{array}{cccccccc}
\ddots & \ddots & \ddots & & & & 0 \\
& \check{B}_{N M l} & 0 & \check{B}_{N M u} & & & \\
& & \check{B}_{N M l} & 0 & \check{B}_{N M u} & & \\
& & & \check{B}_{N M l} & 0 & \check{B}_{N M u} & \\
0 & & & & \ddots & \ddots & \ddots
\end{array}\right]
$$


with the entry matrices $\check{B}_{N M l}$ and $\check{B}_{N M u}$ given by

$$
\check{B}_{N M l}=\underbrace{\left[\begin{array}{cccc}
0 & \hat{B}_{N} & \cdots & \hat{B}_{1} \\
& \ddots & \ddots & \vdots \\
& & \ddots & \hat{B}_{N} \\
0 & & & 0
\end{array}\right]}_{(2 M+1)}, \quad \check{B}_{N M u}=\underbrace{\left[\begin{array}{cccc}
0 & & & 0 \\
\hat{B}_{-N} & \ddots & & \\
\vdots & \ddots & \ddots & \\
\hat{B}_{-1} & \cdots & \hat{B}_{-N} & 0
\end{array}\right]}_{(2 M+1)} .
$$

The matrix $\underline{C}_{[N, M]}$ is defined similarly but in terms of $\left\{\hat{C}_{m}\right\}_{m=-N}^{N}$.

Furthermore, by the structure of $\underline{\hat{B}}_{[N, M]}$, it is easy to see that $\left\|\underline{\hat{B}}_{[N, M]}\right\|_{l_{2} / l_{2}} \leq$ $\sum_{|m| \leq N}\left\|\hat{B}_{m}\right\| \leq \sum_{m=-\infty}^{+\infty}\left\|\hat{B}_{m}\right\| \leq K_{B}<\infty$ as in (B.16). This, together with the fact that $\left\|\underline{\hat{C}}_{[N]}\right\|_{l_{2} / l_{2}} \leq K_{C}$, implies that to complete the proof of (B.20) via (B.21), it remains to show that as $M \rightarrow \infty$

$$
\left\|\check{C}_{[N, M]}(\underline{E}(s)-\underline{Q})^{-1}\right\|_{2} \rightarrow 0, \quad\left\|(\underline{E}(s)-\underline{Q})^{-1} \underline{\underline{B}}_{[N, M]}\right\|_{2} \rightarrow 0
$$

uniformly for $s \in \partial \Omega$. Now we show that the convergence of (B.22) is true. To see this, we note that

$$
\begin{aligned}
& \left\|(\underline{E}(s)-\underline{Q})^{-1} \underline{B}_{[N, M]}\right\|_{2} \\
& \quad \leq \|\left(\underline{E}(s)-\underline{Q}^{-1} \underline{\check{B}}_{[N, M]}\left\|_{2}+\right\|(\underline{E}(s)-\underline{Q})^{-1} \underline{\check{B}}_{u[N, M]} \|_{2},\right.
\end{aligned}
$$

where $\underline{B}_{l[N, M]}$ and ${\underline{B_{u}}}_{[N, M]}$ are the lower and upper triangle portions of $\underline{B}_{[N, M]}$,

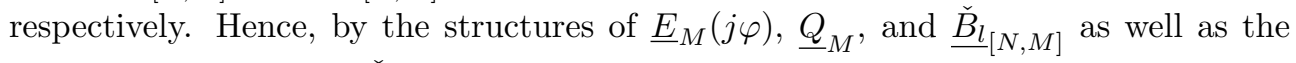
fact that the entries of $\check{B}_{N M l}$ are zero except its right-upper blocks, we have

$$
\begin{aligned}
& \left\|(\underline{E}(s)-\underline{Q})^{-1} \underline{\check{B}}_{[N, M]}\right\|_{2}=\left[\sum_{m}\left\|\left(E_{M m}(s)-Q_{M}\right)^{-1} \check{B}_{N M l}\right\|_{2}^{2}\right]^{1 / 2} \\
& \leq\left[\sum_{m}\left\|\partial_{N}\left(\left(E_{M m}(s)-Q_{M}\right)^{-1}\right)\right\|_{2}^{2} \cdot\left\|\check{B}_{N M l}\right\|^{2}\right]^{1 / 2}
\end{aligned}
$$

where $\partial_{N}(\cdot)$ means taking out the first $N$ block columns from $(\cdot)$. Moreover, by similar arguments to the above, it readily follows that $\left\|\check{B}_{N M l}\right\| \leq K_{B}$ since $\check{B}_{N M l}$ is a submatrix of $\underline{\hat{B}}_{[N, M]}$. Hence, it is easy to see by (B.3) that under our standing assumption $M \geq N+1$,

$$
\begin{aligned}
& \left\|(\underline{E}(s)-\underline{Q})^{-1} \underline{\check{B}}_{l}[N, M]\right\|_{2} \\
& \leq K_{B}\left[\sum_{m} \sum_{k=0}^{N-1} n\left\|((s+j(m(2 M+1)-M+k)) I-Q)^{-1}\right\|_{2}^{2}\right]^{1 / 2} \\
& \leq K_{B} \sqrt{n}\left[\sum_{m} \sum_{k=0}^{N-1} K^{2} f^{2}(m(2 M+1)-M+k)\right]^{1 / 2} \\
& \leq K_{B} \sqrt{n}\left[N K^{2} \sum_{m} \max _{k \in\{0,1, \ldots, N-1\}}\left\{f^{2}(m(2 M+1)-M+k)\right\}\right]^{1 / 2}
\end{aligned}
$$


642

$$
\begin{aligned}
& \leq K K_{B} \sqrt{n N}\left[\sum_{m} f^{2}\left(\min _{k \in\{0,1, \ldots, N-1\}}\{|m(2 M+1)-M+k|\}\right)\right]^{1 / 2} \\
& <K K_{B} \sqrt{n N}\left[\sum_{m} f^{2}(m(M+1))\right]^{1 / 2} \\
& =K K_{B} \sqrt{n N}\left[\frac{1}{(M+1)^{2}} \sum_{m} f^{2}(m)\right]^{1 / 2} \leq \frac{K K_{B} \sqrt{5 n N}}{M+1}
\end{aligned}
$$

Thus, for each fixed $N$ and for any $\mu>0$, there exists an integer $M_{0}^{\prime}(N, \mu)>0$ such that

$$
\left\|\underline{\hat{C}}_{[N]}\right\|_{l_{2} / l_{2}}\left\|(\underline{E}(s)-\underline{Q})^{-1} \underline{\underline{B}}_{[N, M]}\right\|_{2}<\frac{\mu}{4} \quad\left(\forall M \geq M_{0}^{\prime}(N, \mu) \quad \forall s \in \partial \Omega\right) .
$$

The above arguments can be repeated on the second term of the right-hand side of (B.23). Hence, for the same $\mu>0$ and $M_{0}^{\prime}(N, \mu)$, it is easy to see that

$$
\left\|\underline{\hat{C}}_{[N]}\right\|_{l_{2} / l_{2}}\left\|(\underline{E}(s)-\underline{Q})^{-1}{\underline{\check{B}_{u}}}_{[N, M]}\right\|_{2}<\frac{\mu}{4} \quad\left(\forall M \geq M_{0}^{\prime}(N, \mu) \quad \forall s \in \partial \Omega\right),
$$

where we used the fact that $\left\|\check{B}_{N M l}\right\|$ and $\left\|\check{B}_{N M u}\right\|$ have the same upper bound. From (B.26) and (B.28), it follows that

$$
\left\|\underline{\hat{C}}_{[N]}\right\|_{l_{2} / l_{2}}\left\|(\underline{E}(s)-\underline{Q})^{-1} \underline{\underline{B}}_{[N, M]}\right\|_{2}<\frac{\mu}{2} \quad\left(\forall M \geq M_{0}^{\prime}(N, \mu) \quad \forall s \in \partial \Omega\right) .
$$

In a similar way, one can conclude that for each fixed $N$ and any $\mu>0$, there exists an integer $M_{0}^{\prime \prime}(N, \mu)>0$ such that

$$
\left\|\underline{\hat{B}}_{[N, M]}\right\|_{l / 2 l_{2}}\left\|\underline{\check{C}}_{[N, M]}(\underline{E}(s)-\underline{Q})^{-1}\right\|_{2}<\frac{\mu}{2} \quad\left(\forall M \geq M_{0}^{\prime \prime}(N, \mu) \quad \forall s \in \partial \Omega\right) .
$$

Then, the desired convergence assertion follows from (B.21), (B.28), and (B.29) by taking $M_{0}(N, \mu)=\max \left\{M_{0}^{\prime}(N, \mu), M_{0}^{\prime \prime}(N, \mu)\right\}$.

Proof of Lemma 4.4. To see the first assertion of (4.8), we observe from (B.3) that

$$
\begin{aligned}
\left\|G_{m[N, M]}(s)\right\| \leq\left\|\hat{C}_{N M}\right\| \cdot\left\|\left(E_{M m}(s)-Q_{M}\right)^{-1}\right\| \cdot\left\|\hat{B}_{N M}\right\| \\
\leq K\left\|\hat{C}_{N M}\right\| \cdot\left\|\hat{B}_{N M}\right\| \max \{f(m(2 M+1)-M), \ldots, \\
\quad f(m(2 M+1)), \ldots, f(m(2 M+1)+M)\} \\
<K\left\|\hat{C}_{N M}\right\| \cdot\left\|\hat{B}_{N M}\right\| f(|m|(M+1)) \leq K K_{B}^{\prime} K_{C}^{\prime} f(|m|(M+1))
\end{aligned}
$$

for each $|m| \geq 1$. Here $\left\|\hat{C}_{N M}\right\|$ and $\left\|\hat{B}_{N M}\right\|$ have upper bounds independent of $N$ and $M$ (see similar arguments around (B.16)), denoted by $K_{B}^{\prime}$ and $K_{C}^{\prime}$. Inequality (B.30) says that for each fixed $N$, there exists a large enough integer $M_{0}$ with $M_{0} \geq$ $N+1$ such that $\left\|G_{m[N, M]}(s)\right\|<1$ for all $|m| \geq 1, M \geq M_{0}$, and $s \in \partial \Omega$. Thus, $\left|\lambda_{k}\left(G_{m[N, M]}(s)\right)\right|<1$, from which Lemma 15.8 of [24] and (B.30) yield that

$$
\begin{aligned}
& \sum_{m \neq 0} \sum_{k}\left|1-\left(1+\lambda_{k}\left(G_{m[N, M]}(s)\right)\right) \exp \left\{-\lambda_{k}\left(G_{m[N, M]}(s)\right)\right\}\right| \\
& \quad \leq \sum_{m \neq 0} \sum_{k}\left[K K_{B}^{\prime} K_{C}^{\prime} f(|m|(M+1))\right]^{2} \\
& \quad=\left[K K_{B}^{\prime} K_{C}^{\prime}\right]^{2} \frac{(2 M+1) d_{C}}{(M+1)^{2}} \sum_{m \neq 0} f^{2}(m)<\frac{8 d_{C}\left[K K_{B}^{\prime} K_{C}^{\prime}\right]^{2}}{M+1},
\end{aligned}
$$


where $d_{C}$ is the row dimension of the output matrix $C(t)$ of the FDLCP system (2.1).

On the other hand, it is straightforward to show by induction that

$$
\left|\prod_{k}\left(1+a_{k}\right)-1\right| \leq \exp \left\{\sum_{k}\left|a_{k}\right|\right\}-1,
$$

where $a_{k} \in \mathbf{C}$. Since $\sum_{m \neq 0} \sum_{k}\left|1-\left(1+\lambda_{k}\left(G_{m[N, M]}(s)\right)\right) \exp \left\{-\lambda_{k}\left(G_{m[N, M]}(s)\right)\right\}\right| \rightarrow 0$ as $M \rightarrow \infty$ by (B.31), it follows from (B.32) that

$$
\prod_{m \neq 0} \prod_{k}\left(1+\lambda_{k}\left(G_{m[N, M]}(s)\right)\right) \exp \left\{-\lambda_{k}\left(G_{m[N, M]}(s)\right)\right\} \rightarrow 1
$$

uniformly with respect to $s$. This gives the first assertion.

To see the second assertion of (4.8), we observe

$$
\begin{aligned}
\left\|\tilde{\Delta}_{m[N, M]}(s+\rho)\right\| & \leq\left\|E_{M m}^{-1}(s+\rho)\right\|\left\|Q_{M}+\rho I_{M}\right\|\left\|\left(E_{M m}(s)-Q_{M}\right)^{-1}\right\|\left\|\widehat{B C}_{N M}\right\| \\
\text { B.33) } & <K K_{\varphi} f^{2}(|m|(M+1))(\|Q\|+\rho)\left\|\widehat{B C}_{N M}\right\|
\end{aligned}
$$

for each $|m| \geq 1$. In the derivation of (B.33), we repeated some arguments similar to those in (B.30) and used the fact that $\left\|E_{M m}^{-1}(s+\rho)\right\| \leq K_{\varphi} f(|m|(2 M+1)-M)<$ $K_{\varphi} f(|m|(M+1))$. It is easy to see that under the given assumptions about the system matrices, $\left\|\widehat{B C}_{N M}\right\|$ has an upper bound independent of $N$ and $M$, denoted by $K_{B C}$. Then it follows that

$$
\begin{aligned}
& \left|\sum_{m \neq 0} \operatorname{tr}\left(\tilde{\Delta}_{m[N, M]}(s+\rho)\right)\right| \leq \sum_{m \neq 0}(2 M+1) n\left\|\tilde{\Delta}_{m[N, M]}(s+\rho)\right\| \\
& \quad<\sum_{m \neq 0}(2 M+1) n K K_{\varphi} K_{B C} f^{2}(|m|(M+1))(\|Q\|+\rho) \\
& <\frac{4(2 M+1) n K K_{\varphi} K_{B C}(\|Q\|+\rho)}{(M+1)^{2}} \rightarrow 0 \quad(M \rightarrow \infty)
\end{aligned}
$$

which leads to the desired assertion. This completes the proof.

Proof of (4.9). By the det $_{2}$ definition and the block-diagonal structure of the infinite-dimensional matrix $\underline{G}_{[N, M]}(s)$, it is evident that

$$
\begin{aligned}
\mid \operatorname{det}_{2}[ & {\left[\underline{I}+\underline{G}_{[N, M]}(s)\right] \exp \left\{-\tilde{\Delta}_{[N, M]}(s+\rho)\right\} } \\
& -\operatorname{det}_{2}\left[I_{M}+G_{0[N, M]}(s)\right] \exp \left\{-\operatorname{tr}\left(\tilde{\Delta}_{0[N, M]}(s+\rho)\right)\right\} \mid \\
\leq & \left|\operatorname{det}_{2}\left[I_{M}+G_{0[N, M]}(s)\right] \exp \left\{-\operatorname{tr}\left(\tilde{\Delta}_{0[N, M]}(s+\rho)\right)\right\}\right| \\
\times & {\left[\left|\prod_{m \neq 0} \prod_{k}\left(1+\lambda_{k}\left(G_{0[N, M]}(s)\right)\right) \exp \left\{-\lambda_{k}\left(G_{0[N, M]}(s)\right)\right\}-1\right|\right.} \\
& \times\left|\exp \left\{-\sum_{m \neq 0} \operatorname{tr}\left(\tilde{\Delta}_{m[N, M]}(s+\rho)\right)\right\}\right| \\
& \left.+\left|\exp \left\{-\sum_{m \neq 0} \operatorname{tr}\left(\tilde{\Delta}_{m[N, M]}(s+\rho)\right)\right\}-1\right|\right]
\end{aligned}
$$

This, together with Lemma 4.4, tells us that (4.9) follows if it is shown that $\mid \operatorname{det}_{2}\left[I_{M}+\right.$ $\left.G_{0[N, M]}(s)\right] \mid$ and $\left|\operatorname{tr}\left(\tilde{\Delta}_{0[N, M]}(s+\rho)\right)\right|$ are uniformly bounded over $M$ and $s \in \partial \Omega$. 
To see the uniform boundedness of $\left|\operatorname{tr}\left(\tilde{\Delta}_{0[N, M]}(s+\rho)\right)\right|$, we observe by $(4.5)$ that

$$
\begin{aligned}
\mid & \operatorname{tr} \\
& \left(\tilde{\Delta}_{0[N, M]}(s+\rho)\right) \mid \leq\left\|\tilde{\Delta}_{0[N, M]}(s+\rho)\right\|_{1} \\
& \leq\left\|E_{M 0}^{-1}(s+\rho)\left(Q_{M}+\rho I_{M}\right)\right\|_{2}\left\|\left(E_{M 0}(s)-Q_{M}\right)^{-1} \widehat{\underline{B C}}_{[N, M]}\right\|_{2} \\
& \leq\left\|E_{M 0}^{-1}(s+\rho)\right\|_{2}\left\|Q_{M}+\rho I_{M}\right\| \cdot\left\|\left(E_{M 0}(s)-Q_{M}\right)^{-1}\right\|_{2}\left\|\widehat{B C}_{[N, M]}\right\| \\
& \leq(\|Q\|+\rho)\left\|\underline{B C}_{[N, M]}\right\| \sum_{|i| \leq M} n K_{\varphi}^{2} f^{2}(i) \sum_{|i| \leq M} n K^{2} f^{2}(i) \\
& <\left(5 n K K_{\varphi}\right)^{2}(\|Q\|+\rho)\left\|\widehat{B C}_{[N, M]}\right\| .
\end{aligned}
$$

Hence, the desired uniform boundedness follows from the fact that $\left\|\widehat{\widehat{B C}}_{[N, M]}\right\|$ has an upper bound independent of $M$.

To see the uniform boundedness of $\left|\operatorname{det}_{2}\left[I_{M}+G_{0[N, M]}(s)\right]\right|$, we need some preparations. By Theorem 7.4 of [11, p. 69], we have

$$
\left|\operatorname{det}_{2}\left[I_{M}+G_{0[N, M]}(s)\right]\right| \leq \exp \left\{\frac{1}{2}\left\|G_{0[N, M]}(s)\right\|_{2}\right\}
$$

which implies that to see the uniform boundedness of $\left|\operatorname{det}_{2}\left[I_{M}+G_{0[N, M]}(s)\right]\right|$, it suffices to show that $\left\|G_{0[N, M]}(s)\right\|_{2}$ is uniformly bounded over $M$ and $s \in \partial \Omega$. To see this, we note that $\hat{B}_{N M}$ and $\hat{C}_{N M}$ are submatrices of $\underline{\hat{B}}_{[N]}$ and $\underline{\hat{C}}_{[N]}$, respectively. Hence, we immediately have

$$
\begin{aligned}
\left\|G_{0[N, M]}(s)\right\|_{2} & \leq\left\|\hat{C}_{N M}\right\| \cdot\left\|\left(E_{M 0}(s)-Q_{M}\right)^{-1}\right\|_{2} \cdot\left\|\hat{B}_{N M}\right\| \\
& \leq K_{C} K_{B} \sum_{|i| \leq M} n K^{2} f^{2}(i)<5 n K^{2} K_{C} K_{B} .
\end{aligned}
$$

This completes the proof.

\section{REFERENCES}

[1] A. Allievi And A. Soudack, Ship stability via the Mathieu equation, Internat. J. Control, 51 (1990), pp. 139-167.

[2] B. Bamieh And J. B. Pearson, A general framework for linear periodic systems with applications to $H^{\infty}$ sampled-data control, IEEE Trans. Automat. Control, 37 (1992), pp. 418-435.

[3] S. Bittanti and P. Colaneri, Stabilization of periodic systems: Overview and advances, in Proceedings of IFAC Workshop on Periodic Control Systems, Italy, 2001, pp. 157-162.

[4] P. Bolzern and P. Colaneri, The periodic Lyapunov equation, SIAM J. Matrix Anal. Appl., 9 (1988), pp. 499-512.

[5] A. Böttcher and B. Silbermann, Analysis of Toeplitz Operators, Springer-Verlag, Berlin, 1990.

[6] M. Cantoni And K. Glover, Existence of right and left representations of the graph for linear periodically time-varying systems, SIAM J. Control Optim., 38 (2000), pp. 786-802.

[7] J. Dugundu and J. H. Wendell, Some analysis methods for rotating systems with periodic coefficients, AIAA J., 21 (1983), pp. 890-897.

[8] M. Farkas, Periodic Motions, Springer-Verlag, New York, 1994.

[9] I. Gohberg, S. Goldberg, and M. A. Kanshoek, Classes of Linear Operators, Vol. I, Birkhäuser, Basel, 1990.

[10] I. Gohberg, S. Goldberg, and M. A. Kanshoek, Classes of Linear Operators, Vol. II, Birkhäuser, Basel, 1993.

[11] I. Gohberg, S. Goldberg, And N. Krupnik, Traces and Determinants of Linear Operators, Birkhäuser, Basel, 2000. 
[12] J. Guckenheimer and P. Holmes, Nonlinear Oscillations, Dynamical Systems, and Bifurcations of Vector Fields, Springer-Verlag, New York, 1983.

[13] T. Hagiwara, Nyquist stability criterion and positive-realness of sampled-data systems, Systems Control Lett., 45 (2002), pp. 283-291.

[14] A. Halanay, Differential Equations: Stability, Oscillations, Time Lags, Academic Press, New York, 1966.

[15] S. R. Hall and N. M. Wereley, Generalized Nyquist criterion for linear time systems, in Proceedings of American Control Conference, San Diego, 1990, pp. 1518-1525.

[16] C. Y. KaO, A. Megretski, and U. T. Jönsson, A cutting plane algorithm for robustness analysis of periodically time-varying systems, IEEE Trans. Automat. Control, 46 (2001), pp. 579-592.

[17] G. A. Korn And T. M. Korn, Mathematical Handbook for Scientist and Engineers, 2nd ed., McGraw-Hill, New York, 1968.

[18] D. L. Lukes, Differential Equations: Classical to Controlled, Academic Press, New York, 1982.

[19] P. Montagnier, R. J. Spiteri, and J. Angeles, The control of linear time-periodic systems using Floquet-Lyapunov theory, Internat. J. Control, 77 (2004), pp. 472-490.

[20] A. H. Nayfeh and D. T. Mook, Nonlinear Oscillations, John Wiley and Sons, New York, 1979.

[21] A. W. Naylor And G. R. Sell, Linear Operator Theory in Engineering and Science, SpringerVerlag, New York, 1982.

[22] M. Pavella and P. G. Murthy, Transient Stability of Power System-Theory and Practice, John Wiley and Sons, New York, 1994.

[23] J. A. Richards, Analysis of Periodically Time-Varying System, Springer-Verlag, New York, 1983.

[24] W. Rudin, Real and Complex Analysis, 3rd ed., McGraw-Hill, New York, 1987.

[25] J. J. Stoker, Nonlinear Vibrations in Mechanical and Electrical Systems, Interscience Publishers, New York, 1950.

[26] N. M. Wereley, Analysis and Control of Linear Periodically Time Varying Systems, Ph.D. thesis, Department of Aeronautics and Astronautics, M.I.T., 1990.

[27] V. A. Yakubovich and V. M. Starzhinskit, Linear Differential Equations with Periodic Coefficients, Vol. I, John Wiley and Sons, New York, 1975.

[28] V. A. Yakubovich, Dichotomy and absolute stability of nonlinear systems with periodically non-stationary linear part, Systems Control Lett., 11 (1988), pp. 221-228.

[29] J. Zhou AND T. Hagiwara, Existence conditions and properties of frequency response operators of continuous-time periodic systems, SIAM J. Control Optim., 40 (2002), pp. 1867-1887.

[30] J. ZHOU AND T. HAGIWARA, $H_{2}$ and $H_{\infty}$ norm computations of linear continuous-time periodic systems via the skew analysis of frequency response operators, Automatica, 38 (2002), pp. 1381-1387.

[31] J. Zhou, T. Hagiwara, AND M. Araki, Stability analysis of continuous-time periodic systems via the harmonic analysis, IEEE Trans. Automat. Control, 47 (2002), pp. 292-298.

[32] J. ZHou, Harmonic Analysis of Linear Continuous-Time Periodic Systems, Ph.D. thesis, Department of Electrical Engineering, Kyoto University, Kyoto, Japan, 2002. 